\title{
Primordial Non-Gaussianity in the Cosmic Microwave Background
}

\author{
Amit P. S. Yadav ${ }^{1}$ and Benjamin D. Wandelt ${ }^{2,3}$ \\ ${ }^{1}$ Institute for Advanced Study, School of Natural Sciences, Einstein Drive, Princeton, NJ 08540, USA \\ ${ }^{2}$ Department of Astronomy and Physics, University of Illinois at Urbana-Champaign, Urbana, IL 61801, USA \\ ${ }^{3}$ Institut d'Astrophysique de Paris, 98 bis, Boulevard Arago, 75014 Paris, France
}

Correspondence should be addressed to Amit P. S. Yadav, ayadav@ias.edu

Received 17 March 2010; Accepted 3 June 2010

Academic Editor: Eiichiro Komatsu

Copyright (c) 2010 A. P. S. Yadav and B. D. Wandelt. This is an open access article distributed under the Creative Commons Attribution License, which permits unrestricted use, distribution, and reproduction in any medium, provided the original work is properly cited.

In the last few decades, advances in observational cosmology have given us a standard model of cosmology. We know the content of the universe to within a few percent. With more ambitious experiments on the way, we hope to move beyond the knowledge of what the universe is made of, to why the universe is the way it is. In this paper we focus on primordial non-Gaussianity as a probe of the physics of the dynamics of the universe at the very earliest moments. We discuss (1) theoretical predictions from inflationary models and their observational consequences in the cosmic microwave background (CMB) anisotropies; (2) CMBbased estimators for constraining primordial non-Gaussianity with an emphasis on bispectrum templates (3) current constraints on non-Gaussianity and what we can hope to achieve in the near future and (4) nonprimordial sources of non-Gaussianities in the $\mathrm{CMB}$ such as bispectrum due to second order effects, three way crosscorrelation between primary-lensing-secondary $\mathrm{CMB}$, and possible instrumental effects.

\section{Motivation}

In the last few decades the advances in observational cosmology have led the field to its "golden age." Cosmologists are beginning to nail down the basic cosmological parameters. We now know that we live in a Universe which is $13.7 \pm$ $0.1 \mathrm{Gyr}$ old and is spatially flat to about $1 \%$ and is made of $4.6 \pm 0.1 \%$ baryons, $22.8 \pm 1.3 \%$ dark matter, and remaining $72.6 \pm 1.5 \%$ in the form of dark energy. Although we know the constituents to high accuracy, we still do not completely understand the physics of the beginning, the nature of dark energy and dark matter. Many upcoming CMB experiments complimented with observational campaign to map 3D structure of the Universe and new particle physics constraints from the Large Hadron Collider will enable us to move beyond the knowledge of what the universe is made of, to why the universe is the way it is. In this paper we focus on learning about the physics responsible for the initial conditions for the universe.

Inflation [1-4] is perhaps one of the most promising paradigms for the early universe, which, apart from solving some of the problems of the Big Bang model like the flatness and horizon problem, also gives a mechanism for producing the seed perturbations for structure formation [5-9] and other testable predictions.

Most observational probes based on 2-point statistics like $\mathrm{CMB}$ power spectrum still allow vast number of inflationary models. Moreover, the alternatives to inflation such as cyclic models are also compatible with the data. Characterizing the non-Gaussianity in the primordial perturbations has emerged as powerful probe of the early universe. The amplitude of non-Gaussianity is described in terms of dimensionless nonlinearity parameter $f_{\mathrm{NL}}$ (defined in Section 3). Different models of inflation predict different amounts of $f_{\mathrm{NL}}$, starting from $O(1)$ to $f_{\mathrm{NL}} \sim 100$, above which values have been excluded by the WMAP data already. Non-Gaussianity from the simplest inflation models that are based on a slowly rolling scalar field is very small [10-15]; however, a very large class of more general models with, for example, multiple scalar fields, features in inflaton potential, nonadiabatic fluctuations, noncanonical kinetic terms, deviations from Bunch-Davies vacuum, among others [16], for 
a review and references therein generates substantially higher amounts of non-Gaussianity.

The measurement of the bispectrum of the CMB anisotropies is one of the most promising and "clean" way of constraining $f_{\mathrm{NL}}$. Many efficient methods for evaluating bispectrum of CMB temperature anisotropies exist [17-21]. So far, the bispectrum tests of non-Gaussianity have not detected any significant $f_{\mathrm{NL}}$ in temperature fluctuations mapped by COBE [22] and WMAP [18, 23-28]. On the other hand, some authors have claimed non-Gaussian signatures in the WMAP temperature data [29-33]. These signatures cannot be characterized by $f_{\mathrm{NL}}$ and are consistent with nondetection of $f_{\mathrm{NL}}$.

Currently the constraints on the $f_{\mathrm{NL}}$ come from temperature anisotropy data alone. By also having the polarization information in the cosmic microwave background, one can improve sensitivity to primordial fluctuations $[34,35]$. Although the experiments have already started characterizing polarization anisotropies [36-39], the errors are large in comparison to temperature anisotropy. The upcoming experiments such as Planck will characterize polarization anisotropy to high accuracy.

The organization of the paper is as follow. In Section 2 we review the inflationary cosmology focusing on how the microscopic quantum fluctuations during inflation get converted into macroscopic sees perturbations for structure formation, and as CMB anisotropies. In Section 3 we discuss theoretical predictions for non-Gaussianity from the inflationary cosmology. In Section 4 we show how the primordial non-Gaussianity is connected to the CMB bispectrum, and describe/review CMB bispectrum-based estimators to constrain primordial non-Gaussianity $\left(f_{\mathrm{NL}}\right)$. In Section 5 we discuss the current constraints on $f_{\mathrm{NL}}$ by CMB bispectrum and what we can hope to achieve in near future. We also discuss non-primordial sources of non-Gaussianity which contaminate primordial bispectrum signal. In Section 6 we discuss other methods for constraining $f_{\mathrm{NL}}$ besides CMB bispectrum. Finally in Section 7 we summarize with concluding remarks.

\section{Introduction: The Early Universe}

One of the most promising paradigms of the early universe is inflation $[1,2,4]$, which, apart from solving the flatness, homogeneity, and isotropy problem, also gives a mechanism for producing the seed perturbations for structure formation and other testable predictions ${ }^{1}$ (for a recent review of inflationary cosmology see [40]). During inflation, the universe goes through an exponentially expanding phase. From the Friedman equation, the condition for the accelerated expansion is

$$
\rho+3 p<0
$$

For both matter and radiation this condition is not satisfied. But it turns out that for a scalar field, the above condition

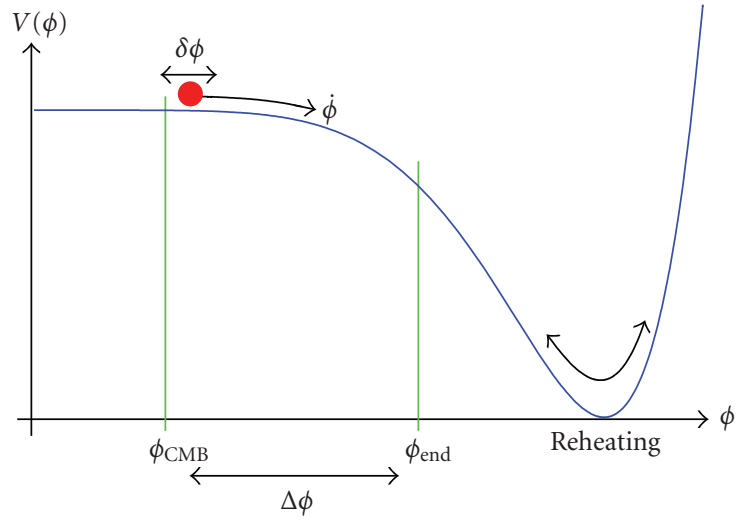

Figure 1: A toy scenario for the dynamics of the scalar field during inflation. During the flat part of potential, universe expand exponentially. When field reaches near the minima of the potential, the field oscillates and the radiation is generated.

can be achieved. For a spatially homogeneous scalar field, $\phi$, moving in a potential, $V(\phi)$, the energy density is given by

$$
\rho_{\phi}=\frac{1}{2}\left(\frac{d \phi}{d t}\right)^{2}+V(\phi)
$$

and the pressure is given by

$$
p_{\phi}=\frac{1}{2}\left(\frac{d \phi}{d t}\right)^{2}-V(\phi)
$$

Hence the condition for accelerated expansion of the universe dominated with scalar field $\phi$ is

$$
\left(\frac{d \phi}{d t}\right)^{2}<V(\phi)
$$

Physically this condition corresponds to situations where kinetic energy of the field is much smaller than its potential energy. This condition is referred to slowly-rolling of the scalar field. During such slow-roll, the Hubble parameter, $H(t)=d \ln a / d t$, is nearly constant in time, and the expansion scale factor $a(t)$ is given by

$$
a(t)=a\left(t_{0}\right) \exp \left(\int_{t_{0}}^{t} H\left(t^{\prime}\right) d t^{\prime}\right) \approx a\left(t_{0}\right) \exp \left[H(t)\left(t-t_{0}\right)\right]
$$

This exponential expansion drives the observable universe spatially flat, homogeneous, and isotropic.

A toy model is shown in Figure 1. In the slow-roll phase, $\phi$ rolls down on $V(\phi)$ slowly, satisfying (4) and hence driving the universe to expand exponentially. Near the minima of the potential, $\phi$ oscillates rapidly and inflation ends. After inflation ends, interactions of $\phi$ with other particles lead $\phi$ to decay with a decay rate of $\Gamma_{\phi}$, producing particles and radiation. This is called a reheating phase of the universe, as $\phi$ converts its energy density into heat by the particle production. 
Not only does inflation solve, the flatness, homogeneity and isotropy problem, but it also gives a mechanism for generating seed perturbations. During inflation the quantum fluctuation in the field $\phi$ exponentially stretched due to the rapid expansion phase. The proper wavelength of the fluctuations stretched out of the Hubble-horizon scale to that time, $H^{-1}$. Once outside the horizon, the characteristic rms amplitude of these fluctuations is $|\phi|_{\mathrm{rms}} \sim H /(2 \pi)$. These fluctuations do not change in time while outside the horizon. After inflation, and reheating, the standard hot-big scenario starts. As the universe decelerates, at some point the fluctuations reenter the Hubble horizon, seeding matter and radiation fluctuations in the universe. Figure 2 summarizes the evolution of characteristic length scales.

2.1. Primordial Perturbations. We use linearly perturbed conformal Friedmann Lematre Robertson Walker (FLRW) metric of the form

$$
\begin{aligned}
d s^{2}=a^{2}(\tau)\{ & -(1+2 A Q) d \tau^{2}-2 B Q_{i} d \tau d x^{i} \\
& \left.+\left[\left(1+2 H_{\mathrm{L}} Q\right) \delta_{i j}+2 H_{\mathrm{T}} Q_{i j}\right] d x^{i} d x^{j}\right\},
\end{aligned}
$$

where all the metric perturbations, $A, B, H_{\mathrm{L}}$, and $H_{\mathrm{T}}$, are $\ll 1$, and functions of conformal time $\tau$. The spatial coordinate dependence of the perturbations is described by the scalar harmonic eigenfunctions, $Q, Q_{i}$, and $Q_{i j}$, that satisfy $\delta^{i j} Q_{i j}=$ $-k^{2} Q, Q_{i}=-k^{-1} Q_{i}$, and $Q_{i j}=k^{-2} Q_{i j}+(1 / 3) \delta_{i j} Q$. Note that $Q_{i j}$ is traceless: $\delta^{i j} Q_{i j}=0$.

Let us consider two new perturbation variables $[8,41]$;

$$
\begin{gathered}
u \equiv \delta \phi-\frac{\dot{\phi}}{a H} \mathcal{R}, \\
\zeta \equiv-\frac{a H}{\dot{\phi}} u=\mathcal{R}-\frac{a H}{\dot{\phi}} \delta \phi,
\end{gathered}
$$

which are Gauge invariant. Here $\mathcal{R} \equiv H_{\mathrm{L}}+(1 / 3) H_{\mathrm{T}}$ is perturbations in the intrinsic spatial curvature. While $u$ reduces to $\delta \phi$ in the spatially flat Gauge $(\mathcal{R} \equiv 0)$, or to $-(\dot{\phi} / a H) \mathcal{R}$ in the comoving gauge $(\delta \phi \equiv 0)$, its value is invariant under any gauge transformation. Similarly $\zeta$, which reduces to $\mathcal{R}$ in the comoving gauge, and to $-(a H / \dot{\phi}) \delta \phi$ in the spatially flat gauge, is also gauge invariant. The perturbation variable $\zeta$ helps the perturbation analysis not only because of being gauge invariant, but also because it is conserved on super-horizon scales throughout the cosmic evolution.

The quantum fluctuations generate the gauge-invariant perturbation, $u$, that reduces to either $\delta \phi$ or $(\dot{\phi} / a H) \mathcal{R}$ depending on which gauge we use, either the spatially flat gauge or the comoving gauge. Hence, $\delta \phi_{\text {flat }}$ and $(\dot{\phi} / a H) \mathcal{R}_{\text {com }}$ are equivalent to each other at linear order. The benefit of using $u$ is that it relates these two variables unambiguously, simplifying the transformation between $\delta \phi_{\text {flat }}$ and $\mathcal{R}_{\text {com }}$.

The solution for $\zeta$ is valid throughout the cosmic history regardless of whether a scalar field, radiation, or matter dominates the universe; thus, once created and leaving the

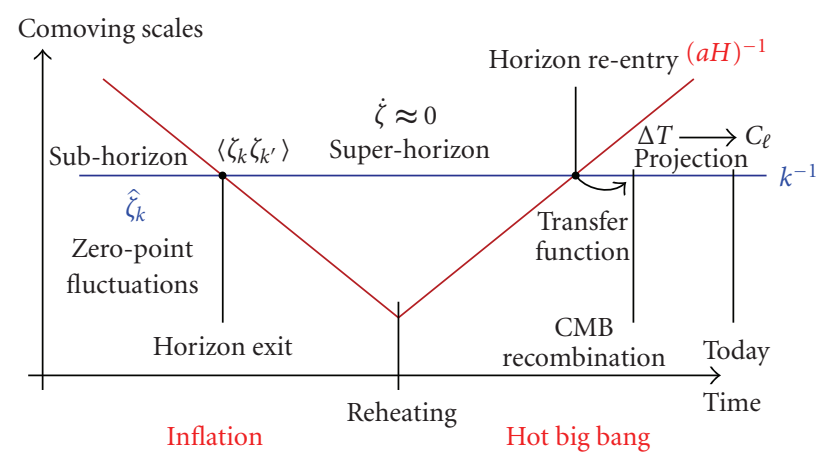

FIGURE 2: Evolution of comoving horizon and generation of perturbations in the inflationary universe. figure is from [40].

Hubble horizon during inflation, $\zeta$ remains constant in time throughout the subsequent cosmic evolution until reentering the horizon. The amplitude of $\zeta$ is fixed by the quantumfluctuation amplitude in $u$

$$
\Delta_{\zeta}^{2}(k)=\left(\frac{a H}{\dot{\phi}}\right)^{2} \Delta_{\phi}^{2}(k) \approx\left(\frac{a H^{2}}{2 \pi \dot{\phi}}\right)^{2}=\left[\frac{H^{2}}{2 \pi\left(\frac{d \phi}{d t}\right)}\right]^{2} .
$$

This is the spectrum of $\zeta$ on super-horizon scales.

2.2. From Primordial Perturbations to $C M B$ Anisotropies. The metric perturbations perturb $\mathrm{CMB}$, producing the $\mathrm{CMB}$ anisotropy on the sky. Among the metric perturbation variables, the curvature perturbations play a central role in producing the $\mathrm{CMB}$ anisotropy.

As we have shown in the previous subsection, the gaugeinvariant perturbation, $\zeta$, does not change in time on superhorizon scales throughout the cosmic evolution regardless of whether a scalar field, radiation, or matter dominates the universe. The intrinsic spatial curvature perturbation, $\mathcal{R}$, however, does change when equation of state of the universe, $w \equiv p / \rho$, changes. Since $\zeta$ remains constant, it is useful to write the evolution of $\mathcal{R}$ in terms of $\zeta$ and $w$; however, $\mathcal{R}$ is not gauge invariant itself, but $\zeta$ is gauge invariant, so that the relation between $\mathcal{R}$ and $\zeta$ may look misleading. In 1980, Bardeen et al. [42] introduced another gauge-invariant variable, $\Phi$ (or $\Phi_{\mathrm{H}}$ in the original notation), which reduces to $\mathcal{R}$ in the zero-shear gauge, or the Newtonian gauge, in which $B \equiv 0 \equiv H_{\mathrm{T}}$. $\Phi$ is given by

$$
\Phi=\mathcal{R}-\frac{a H}{k}\left(-B-\frac{\dot{H}_{\mathrm{T}}}{k}\right) .
$$

Here, the terms in the parenthesis represent the shear, or the anisotropic expansion rate, of the $\tau=$ constant hypersurfaces. While $\Phi$ represents the curvature perturbations in the 
zero-shear gauge, it also represents the shear in the spatially flat gauge in which $\mathcal{R} \equiv 0$. Using $\Phi$, we may write $\zeta$ as

$$
\zeta=\mathcal{R}-\frac{a H}{\dot{\phi}} \delta \phi=\Phi-\frac{a H}{k}\left(v_{\phi}-\frac{\dot{H}_{\mathrm{T}}}{k}\right),
$$

where the terms in the parenthesis represent the gaugeinvariant fluid velocity.

We use $\Phi$ in rest of the paper because it gives the closest analogy to the Newtonian potential, which we have some intuition of. $\Phi$ reduces to $\mathcal{R}$ in the zero-shear gauge (or the Newtonian gauge) in which the metric (6) becomes just like the Newtonian limit of the general relativity.

The gauge-invariant velocity term, $v-k^{-1} \dot{H}_{\mathrm{T}}$, differentiates $\zeta$ from $\Phi$. Since this velocity term depends on the equation of state of the universe, $w=p / \rho$, the velocity and $\Phi$ change as $w$ changes, while $\zeta$ is independent of $w$. The evolution of $\Phi$ on super-horizon scales in cosmological linear perturbation theory gives the following [43]:

$$
\Phi=\frac{3+3 w}{5+3 w} \zeta
$$

for adiabatic fluctuations, and hence $\Phi=(2 / 3) \zeta$ in the radiation era $(w=1 / 3)$, and $\Phi=(3 / 5) \zeta$ in the matter era $(w=0)$. $\Phi$ then perturbs CMB through the so-called (static) Sachs-Wolfe effect [44]

$$
\frac{\Delta T}{T}=-\frac{1+w}{5+3 w} \zeta
$$

At the decoupling epoch, the universe has already been in the matter era in which $w=0$, so that we observe adiabatic temperature fluctuations of $\Delta T / T=-(1 / 3) \Phi=-(1 / 5) \zeta$, and the CMB fluctuation spectrum of the Sachs-Wolfe effect, $\Delta_{\mathrm{SW}}^{2}(k)$, is

$$
\Delta_{\mathrm{SW}}^{2}(k)=\frac{1}{9} \Delta_{\Phi}^{2}(k)=\frac{1}{25} \Delta_{\zeta}^{2}(k) .
$$

By projecting the 3-dimensional CMB fluctuation spectrum, $\Delta_{\mathrm{SW}}^{2}(k)$, on the sky, we obtain the angular power spectrum ${ }^{2}$, $C_{l}[45]$,

$$
\begin{aligned}
C_{l}^{S W} & =4 \pi \int_{0}^{\infty} \frac{d k}{k} \Delta_{S W}^{2}(k) j_{l}^{2}\left[k\left(\tau_{0}-\tau_{\mathrm{dec}}\right)\right] \\
& =C_{2}^{S W} \frac{\Gamma\left[\left(9-n_{s}\right) / 2\right] \Gamma\left[l+\left(n_{s}-1\right) / 2\right]}{\Gamma\left[\left(n_{s}+3\right) / 2\right] \Gamma\left[l+\left(5-n_{s}\right) / 2\right]},
\end{aligned}
$$

where $\tau_{0}$ and $\tau_{\mathrm{dec}}$ denote the conformal time at the present epoch and at the decoupling epoch, respectively, and $n_{s} \equiv 1+$ $\left[d \ln \Delta^{2}(k) / d \ln k\right]$ is a spectral index which is conventionally used in the literature.

On small angular scales $(\ell>10)$, the Sachs-Wolfe approximation breaks down, and the acoustic physics in the photon-baryon fluid system modifies the primordial radiation spectrum [46]. To calculate the anisotropies at all the scales, one has to solve the Boltzmann photon transfer equation together with the Einstein equations. These equations can be solved numerically with the Boltzmann code such as CMBFAST [47]. The CMB power spectrum then can be written as

$$
C_{\ell}=4 \pi \int_{0}^{\infty} \frac{d k}{k} \Delta_{\Phi}^{2}(k) g_{\mathrm{T} \ell}^{2}(k) .
$$

Here $g_{T \ell}(k)$ is called the radiation transfer function, and it contains all the physics which modifies the primordial power spectrum $\Delta_{\Phi}$ to generate $\mathrm{CMB}$ power spectrum $C_{\ell}$. For the adiabatic initial conditions, in the Sachs-Wolfe limit, $g_{\mathrm{Tl}}(k)=-(1 / 3) j_{l}\left[k\left(\tau_{0}-\tau_{\mathrm{dec}}\right)\right]$. Often in the literature power spectrum, $P_{\Phi}(k)$, is used instead of $\Delta_{\Phi}^{2}(k)$. The two are related as $\Delta_{\Phi}^{2}(k)=\left(2 \pi^{2}\right)^{-1} k^{3} P_{\Phi}(k) . \Delta_{\Phi}^{2}(k)$ is called the dimensionless power spectrum.

If $\Phi$ were exactly Gaussian, all the statistical properties of $\Phi$ would be encoded in the two-point function or in $C_{\ell}$ in the spherical harmonic space. Since $\Phi$ is directly related to $\zeta$ through (11), all the information of $\zeta$ is also in-coded in $C_{\ell}$. Although $\zeta$ which is related to a Gaussian variable, $u$, through $\zeta=-(a H / \dot{\phi}) u$, in the linear order $\zeta$ also obeys Gaussian statistics; however the nonlinear relation between $\zeta$ and $u$ makes $\zeta$ (and hence $\Phi$ and CMB anisotropies) slightly nonGaussian. The non-linear relation between $\zeta$ and $\Phi$ is not the only source of non-Gaussianity in the CMB anisotropies. For example, at the second order, the relationship between $\Phi$ and $\Delta T / T$ is also non-linear.

2.3. Probes of the Cosmological Initial Conditions. The main predictions of a canonical inflation model are the following:

(i) spatial flatness of the observable universe,

(ii) homogeneity and isotropy on large angular scales of the observable universe,

(iii) seed scalar and tensor perturbation with primordial density perturbations being

(a) nearly scale invariant,

(b) nearly adiabatic,

(c) very close to Gaussian.

At the time of writing, these predictions are consistent with all current observations. This represents a major success for the inflationary paradigm. On the other hand, the inflationary paradigm can be realized by a large "zoo"3 of models. In addition, somewhat surprisingly, there exist scenarios where the Universe first contracts and then expands (such as the ekpyrotic/cyclic model), which (up to theoretical uncertainties regarding the precise mechanics of the bounce) also reproduce Universes with the properties described above. What we would like to do is to find observables that allows us to distinguish between members of the inflationary zoo. The exciting fact is that upcoming experiments will have the sensitivity to achieve this goal. Tilt and Running: Inflationary models very generically predict a slight deviation from completely flat spectrum. If we write the primordial power spectrum as $\Delta_{\Phi}(k)=A\left(k_{0}\right)\left(k / k_{0}\right)^{n_{s}-1}$, then $n_{s}=1$ correspond to flat spectrum and the quantity $\left|n_{s}-1\right|$ is called 
a tilt, which characterizes the deviation from scale invariant spectrum. Although the deviations from the scale invariance are predicted to be small, the exact amount of deviation depends on the details of the inflationary model. For example in most slow roll models $|n-1|$ is of order $1 / N_{e}$, where $N_{e} \sim 60$ is a number of $e$-folds to the end of inflation. Ghost inflation, however, predicts negligible tilt. Hence characterizing the tilt of the scalar spectral index is a useful probe of the early universe. Currently the most stringent constraints on tilt come from the WMAP 5-year data, $n_{s}=0.960_{-0.013}^{+0.014}[48]$, which already disfavors inflationary models with "blue spectral index" $\left(n_{s}>1\right)$. The $1 \sigma$ error on $n_{s}$ will reduce to $\Delta n_{s}=0.0036$ for upcoming Planck satellite and to $\Delta n_{s}=0.0016$ for futuristic CMBPol-like satellite [49].

Apart from the tilt in the primordial power spectrum, inflationary models also predict $n_{s}$ to be slightly scale dependent. This scale dependence is referred to as "running" of the spectral index $n_{s}$ and is defined as $d n_{s} / d \ln k$. The constraints on the running from the WMAP 5 -year data are $-0.090<d n_{s} / d \ln k<0.0019$ [48]. The $1 \sigma$ error will reduce to $\Delta\left(d n_{s} / d \ln k\right)=0.0052$ for upcoming Planck satellite and to $\Delta\left(d n_{s} / d \ln k\right)=0.0036$ for a fourth-generation satellite such as CMBPol [49].

Primordial Gravitational Waves. Inflation also generates tensor perturbations (gravitational waves), which although small compared to scalar component, are still detectable, in principle. So far primordial gravitational waves have not been detected. There are upper limits on their amplitude; see [50] for a current observational bounds on the level for primordial gravitational waves. Detection of these tensor perturbations or primordial gravitational waves is considered a "smoking gun" for the inflationary scenario. In contrast to inflation, ekpyrotic (cyclic) models predict an amount of gravitational waves that is much smaller than polarized foreground emission would allow us to see even for an ideal CMB experiment. Primordial scalar perturbations create only E-modes of the $\mathrm{CMB}^{4}$, while primordial tensor perturbations generate both parity even E-modes and parity odd B-modes polarization [51-53]. The detection of primordial tensor B-modes in the $\mathrm{CMB}$ would confirm the existence of tensor perturbations in the early universe. This primordial $B$-mode signal is directly related to the Hubble parameter $H$ during inflation, and thus a detection would establish the energy scale at which inflation happened. Various observational efforts are underway to detect such B-mode signal of the CMB [54]. Search for primordial B-modes is challenging. Apart from foreground subtraction challenges, and the challenge of reaching the instrumental sensitivity to detect primordial B-modes, there are several nonprimordial sources such as weak lensing of $\mathrm{CMB}$ by the large-scale structure $[55,56]$, rotation of the $\mathrm{CMB}$ polarization [57], and instrumental systematics that generate B-modes which contaminate the inflationary signal $[58,59]$. The amplitude of gravitational waves is parametrized as the ratio of the amplitude of tensor and scalar perturbations, $r$. The limit from WMAP 5-year data is $r<0.22(2 \sigma)$ [48].
Isocurvature Modes. Inflationary models with a single scalar field predict primordial perturbations to be adiabatic. Hence detection of isocurvature density perturbations is a "smoking gun" for multifield models of inflation. A large number of inflationary models with multiple scalar fields predict some amount of isocurvature modes [60-72]. For example, curvaton models predict the primordial perturbations to be a mixture of adiabatic and isocurvature perturbations. Isocurvature initial conditions specify perturbations in the energy densities of two (or more) species that add up to zero. It does not perturb the spatial curvature of comoving slice (i.e., $\mathcal{R}$ is zero, hence the name isocurvature). In general, there can be four types of isocurvature modes, namely: baryon isocurvature modes, CDM isocurvature modes, neutrino density isocurvature modes, and neutrino velocity isocurvature modes. These perturbations imprint distinct signatures in the $\mathrm{CMB}$ temperature and E-polarization anisotropies [73]. The contribution of isocurvature modes is model dependent, and different models predict different amounts of it. There exists an upper limit on the allowed isocurvature modes using $\mathrm{CMB}$ temperature anisotropies $[74,75]$ a characterization (or detection of any) of isocurvature modes has a potential of discriminating between early Universe models.

Primordial Non-Gaussianity. Canonical inflationary models predict primordials perturbations to be very close to Gaussian [5-9], and any non-Gaussianity predicted by the canonical inflation models is very small $[14,15]$. However models with nonlinearity $[10,13,76]$, interacting scalar fields $[12,77]$, and deviation from ground state $[78,79]$ can generate large non-Gaussian perturbations. The amplitude of the non-Gaussian contribution to the perturbation is often referred to as $f_{\mathrm{NL}}$ even if the nature of the nonGaussianities can be quite different. Different models of inflation predict different amounts of $f_{\mathrm{NL}}$, starting from very close to zero for almost Gaussian perturbations, to $f_{\mathrm{NL}} \approx 100$ for large non-Gaussian perturbations. For example, the canonical inflation models with slow roll inflation, where only a couple of derivatives of potential, are responsible for inflationary dynamics, predict $f_{\mathrm{NL}} \sim$ 0.05 [15]. In models where higher-order derivatives of the potential are important, the value of $f_{\mathrm{NL}}$ varies from $f_{\mathrm{NL}} \sim$ 0.1 where higher order derivatives are suppressed by a low UV cutoff [80] to $f_{\mathrm{NL}} \sim 100$ based on Dirac-Born-Infeld effective action. Ghost inflation, where during inflation, the background has a constant rate of change as opposed to the constant background in conventional inflation, is also capable of giving $f_{\mathrm{NL}} \sim 100$ [81]. The additional field models generating inhomogeneities in nonthermal species [82] can generate $f_{\mathrm{NL}} \sim 5$ [83]; while curvaton models, where isocurvature perturbations in second field during the inflation generate adiabatic perturbations after the inflation, can have $f_{\mathrm{NL}} \sim 10[84]$.

In the following we will see that non-Gaussianity, far from being merely a test of standard inflation, may reveal detailed information about the state and physics of the very early Universe, if it is present at the level suggested by the theoretical arguments above. 


\section{Primordial Non-Gaussianity}

Large primordial non-Gaussianity can be generated if any of the following condition is violated [85].

(i) Single Field. Only one scalar field is responsible for driving the inflation and the quantum fluctuations in the same field is responsible for generating the seed classical perturbations.

(ii) Canonical Kinetic Energy. The kinetic energy of the field is such that the perturbations travel at the speed of light.

(iii) Slow Roll. During inflation phase the field evolves much slowly than the Hubble time during inflation.

(iv) Initial Vacuum State. The quantum field was in the Bunch-Davies vacuum state before the quantum fluctuation were generated.

To characterize the non-Gaussianity one has to consider the higher order moments beyond two-point function, which contains all the information for Gaussian perturbations. The 3-point function which is zero for Gaussian perturbations contains the information about non-Gaussianity. The 3point correlation function of Bardeen's curvature perturbations, $\Phi(k)$, can be simplified using the translational symmetry to give

$\left\langle\Phi\left(\mathbf{k}_{\mathbf{1}}\right) \Phi\left(\mathbf{k}_{\mathbf{2}}\right) \Phi\left(\mathbf{k}_{\mathbf{3}}\right)\right\rangle=(2 \pi)^{3} \delta^{3}\left(\mathbf{k}_{\mathbf{1}}+\mathbf{k}_{\mathbf{2}}+\mathbf{k}_{\mathbf{3}}\right) f_{\mathrm{NL}} \cdot F\left(k_{1}, k_{2}, k_{3}\right)$,

where $F\left(k_{1}, k_{2}, k_{3}\right)$ tells the shape of the bispectrum in momentum space while the amplitude of non-Gaussianity is captured dimensionless non-linearity parameter $f_{\mathrm{NL}}$. The shape function $F\left(k_{1}, k_{2}, k_{3}\right)$ correlates fluctuations with three wave-vectors and form a triangle in Fourier space. Depending on the physical mechanism responsible for the bispectrum, the shape of the 3-point function, $F\left(k_{1}, k_{2}, k_{3}\right)$, can be broadly classified into three classes [86]. The local, "squeezed," non-Gaussianity, where $F\left(k_{1}, k_{2}, k_{3}\right)$ is large for the configurations in which $k_{1} \ll k_{2} \approx k_{3}$. Most of the studied inflationary and Ekpyrotic models produce non-Gaussianity of local shape (e.g., [82, 84, 87-104]). Second, the nonlocal, "equilateral," non-Gaussianity where $F\left(k_{1}, k_{2}, k_{3}\right)$ is large for the configuration when $k_{1} \approx k_{2} \approx k_{3}$. Finally the folded $[105,106]$ shape, where $F\left(k_{1}, k_{2}, k_{3}\right)$ is large for the configurations in which $k_{1} \approx 2 k_{2} \approx 2 k_{3}$. Figure 3 shows these three shapes.

Non-Gaussianity of Local Type. The local form of nonGaussianity may be parametrized in real space as $s^{5}[13,107$, 108]:

$$
\zeta(\mathbf{r})=\zeta_{L}(\mathbf{r})+\frac{3}{5} f_{\mathrm{NL}}\left(\zeta_{L}^{2}(\mathbf{r})-\left\langle\zeta_{L}^{2}(\mathbf{r})\right\rangle\right)
$$

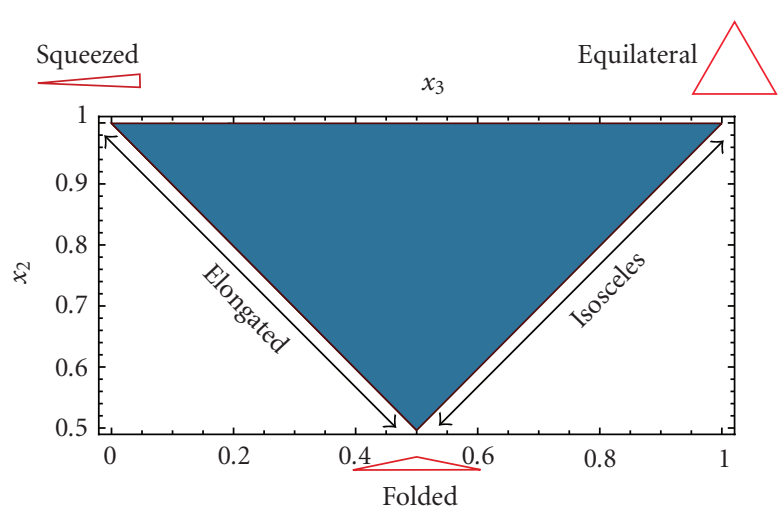

FIGURE 3: Shapes of non-Gaussianity. The shape function $F\left(k_{1}, k_{2}, k_{3}\right)$ forms a triangle in Fourier space. The triangles are parametrized by rescaled Fourier modes, $x_{2}=k_{2} / k_{1}$ and $x_{3}=k_{3} / k_{1}$. Figure is from [40].

where $\zeta_{L}(r)$ is the linear Gaussian part of the perturbations, and $f_{\mathrm{NL}}$ characterizes the amplitude of primordial nonGaussianity. Different inflationary models predict different amounts of $f_{\mathrm{NL}}$, starting from $O(1)$ to $f_{\mathrm{NL}} \sim 100$, beyond which values have been excluded by the Cosmic Microwave Background (CMB) bispectrum of WMAP temperature data. The bispectrum in this model can be written as

$$
\begin{aligned}
& F_{\text {local }}\left(k_{1}, k_{2}, k_{3}\right) \\
& \qquad \begin{aligned}
=2 \Delta_{\Phi}^{2} f_{\mathrm{NL}}[ & \frac{1}{k_{1}^{3-\left(n_{s}-1\right)} k_{2}^{3-\left(n_{s}-1\right)}}+\frac{1}{k_{1}^{3-\left(n_{s}-1\right)} k_{3}^{3-\left(n_{s}-1\right)}} \\
& \left.+\frac{1}{k_{2}^{3-\left(n_{s}-1\right)} k_{3}^{3-\left(n_{s}-1\right)}}\right]
\end{aligned}
\end{aligned}
$$

where $\Delta_{\Phi}$ is the amplitude of the primordial power spectrum.

The local form arises from a non-linear relation between inflaton and curvature perturbations $[10,11,13]$, curvaton models [84], or the New Ekpyrotic models [109, 110]. Models with fluctuations in the reheating efficiency $[9,10]$ and multifield inflationary models [17] also generate nonGaussianity of local type.

Being local in real space, non-Gaussianity of local type describes correlations among Fourier modes of very different $k$. In the limit in which one of the modes becomes of very long wavelength [111], $k_{3} \rightarrow 0$, (i.e., the other two $k^{\prime}$ s become equal and opposite), $\zeta_{\vec{k}_{3}}$ freezes out much before $k_{1}$ and $k_{2}$ and behaves as a background for their evolution. In this limit $F_{\text {local }}$ is proportional to the power spectrum of the short and long wavelength modes

$$
F_{\text {local }} \propto \frac{1}{k_{3}^{3}} \frac{1}{k_{1}^{3}} .
$$


As an example, for canonical single field slow-roll inflationary models, the three-point function is given by [15]

$$
\begin{gathered}
F_{\text {slow-roll }}\left(\vec{k}_{1}, \vec{k}_{2}, \overrightarrow{k_{3}}\right) \\
=\frac{1}{8}\left(\frac{3}{5}\right) \Delta_{\Phi}^{2} \frac{1}{\Pi k_{i}^{3}}\left[(3 \epsilon-2 \eta) \sum_{\vec{k}_{i}} k_{i}^{3}+\epsilon \sum_{i \neq j} k_{i} k_{j}^{2}\right. \\
\left.+8 \epsilon \frac{\sum_{i>j} k_{i}^{2} k_{j}^{2}}{k_{1}+k_{2}+k_{3}}\right],
\end{gathered}
$$

where $\epsilon$ and $\eta$ are the usual slow-roll parameters and are assumed to be much smaller than unity. Taking the limit $k_{3} \rightarrow 0$ gives the local form as in (19). To show this point, Figure 4 compares the non-Gaussianity shape for local type and for slow-roll model. Although in this limit, slow-roll models do predict non-Gaussianity of local type but as evident from (20), the bispectrum of inflaton perturbations yields a non-trivial-scale dependence of $f_{\mathrm{NL}}$ $[12,15]$. However in the slow roll limit $\eta, \epsilon \ll 1$ and hence the amplitude is too small to detect.

Non-Gaussianity of Equilateral Type. While vast numbers of inflationary models predict non-Gaussianity of local type, this model, for instance, fails completely when nonGaussianity is localized in a specific range in $k$ space, the case that is predicted from inflation models with higher derivative terms [ $81,106,112-115]$. In these models the correlation is among modes with comparable wavelengths which go out of the horizon nearly at the same time. The shape function for the equilateral shape can be written as [25]

$$
\begin{aligned}
& F_{\text {equil. }}\left(k_{1}, k_{2}, k_{3}\right) \\
& =f_{\mathrm{NL}}^{\text {equil. }} \cdot 6 \Delta_{\Phi}^{2} \cdot\left[-\frac{1}{k_{1}^{3-\left(n_{s}-1\right)} k_{2}^{3-\left(n_{s}-1\right)}}\right. \\
& +(2 \text { perm. })-\frac{2}{\left(k_{1} k_{2} k_{3}\right)^{2-2\left(n_{s}-1\right) / 3}} \\
& +\frac{1}{k_{1}^{1-\left(n_{s}-1\right) / 3} k_{2}^{2-2\left(n_{s}-1\right) / 3} k_{3}^{3-\left(n_{s}-1\right)}} \\
& +(5 \text { perm. })] .
\end{aligned}
$$

The models of this kind have large $F\left(k_{1}, k_{2}, k_{3}\right)$ for the configurations, where $k_{1} \approx k_{2} \approx k_{3}$. The equilateral form arises from non-canonical kinetic terms such as the DiracBorn-Infeld (DBI) action [112], the ghost condensation [81], or any other single-field models in which the scalar field acquires a low speed of sound $[106,115]$.

As an example, models with higher derivative operators in the usual inflation scenario and a model of inflation based on the Dirac-Born-Infeld (DBI) action produce a bispectrum of the form

$$
\begin{aligned}
& F_{\text {hd }}\left(\vec{k}_{1}, \vec{k}_{2}, \vec{k} 3\right) \\
& =\frac{3}{40} \Delta_{\Phi}^{2} \frac{\dot{\phi}^{2}}{\Lambda^{4}} \frac{1}{\Pi k_{i}^{3}}\left[\frac{1}{k_{1}+k_{2}+k_{3}}\right. \\
& \times\left(\sum_{i} k_{i}^{5}+\sum_{i \neq j}\left(2 k_{i}^{4} k_{j}-3 k_{i}^{3} k_{j}^{2}\right)\right. \\
& \left.\left.+\sum_{i \neq j \neq \ell}\left(k_{i}^{3} k_{j} k_{\ell}-4 k_{i}^{2} k_{j}^{2} k_{\ell}\right)\right)\right] .
\end{aligned}
$$

The previous model uses $\left(1 / 8 \Lambda^{4}\right)(\nabla \phi)^{2}(\nabla \phi)^{2}$ as a leading order operator. DBI inflation, which can produce large nonGaussianity, $f_{\mathrm{NL}} \sim 100$, also has $F\left(k_{1}, k_{2}, k_{3}\right)$ of a similar form.

Ghost inflation, where an inflationary de Sitter phase is obtained with a ghost condensate, produces a bispectrum of the following form [81]:

$$
\begin{aligned}
F_{\text {ghost }}\left(k_{1}, k_{2}, k_{3}\right) & \\
= & -\left(\frac{3}{5}\right)^{3} \cdot \frac{2 \sqrt{2} \pi^{3 / 2}}{\Gamma(1 / 4)^{3}} \frac{H^{5} \beta}{M^{2}}\left(\frac{M}{\alpha H}\right)^{4} \\
& \times \frac{1}{\prod_{i} k_{i}^{3}} \int_{-\infty}^{0} d \eta \eta^{-1} F^{*}(\eta) F^{*}\left(\frac{k_{2}}{k_{1}} \eta\right) F^{*}\left(\frac{k_{3}}{k_{1}} \eta\right) \\
& \times k_{3}\left(\vec{k}_{1} \cdot \vec{k}_{2}\right)+\text { symm. }
\end{aligned}
$$

where $\alpha$ and $\beta$ are free parameters of order unity, and

$$
F(x)=\sqrt{\frac{\pi}{8}}(-x)^{3 / 2} H_{3 / 4}^{(1)}\left(\frac{x^{2}}{2}\right) .
$$

Ghost inflation also produces large non-Gaussianity, $f_{\mathrm{NL}} \sim$ 100. Figure 3 shows the shape of non-Gaussianity of equilateral type by showing $F\left(k_{1}, k_{2}, k_{3}\right)$ for ghost inflation and for a model with a higher derivative term.

Folded Shape. So far the 3-point functions were calculated assuming the regular Bunch-Davis vacuum state, giving rise to either local or equilateral type non-Gaussianity. However if the bispectrum is calculated by dropping the assumption of Bunch-Davis initial state gives rise to bispectrum shape which peaks for the folded shape, $k_{1} \approx 2 k_{2} \approx 2 k_{3}$, with shape function given as $[105,106,116]$

$$
\begin{aligned}
F_{\text {non-BD }} & \left(k_{1}, k_{2}, k_{3}\right)=\left(\frac{3}{5}\right)^{3} \cdot \frac{1}{M_{p}^{2}} \frac{4}{\prod\left(2 k_{i}^{3}\right)} \frac{H^{6}}{\dot{\phi}^{2}} \sum_{j} \frac{3 k_{1}^{2} k_{2}^{2} k_{3}^{2}}{k_{j}^{2} \widetilde{k}_{j}} \\
\times & \operatorname{Re}\left(\beta_{k_{j}}\right)\left(\cos \left(\widetilde{k}_{j} \eta_{0}\right)-1\right),
\end{aligned}
$$




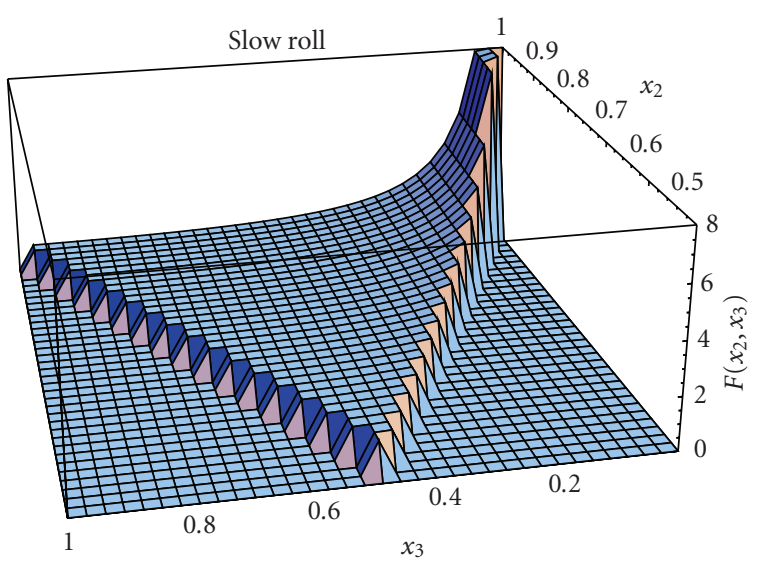

(a)

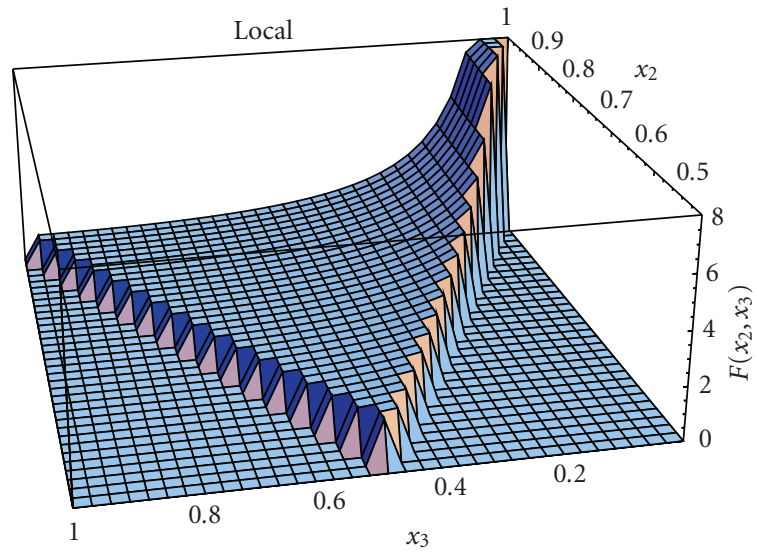

(b)

Figure 4: Plot of the function $F\left(1, x_{2}, x_{3}\right) x_{2}^{2} x_{3}^{2}$ for the Slow-Roll inflation as given by (20) (a) and the local distribution as given by (18) (b). The figures are normalized to have value 1 for equilateral configurations $x_{2}=x_{3}=1$ and set to zero outside the region $1-x_{2} \leq x_{3} \leq x_{2}$. Here $x_{3} \equiv k_{3} / k_{1}, x_{2} \equiv k_{2} / k_{1}$, and $\epsilon=\eta=1 / 30$. The figures are taken from Babich et al. 2004 [86].

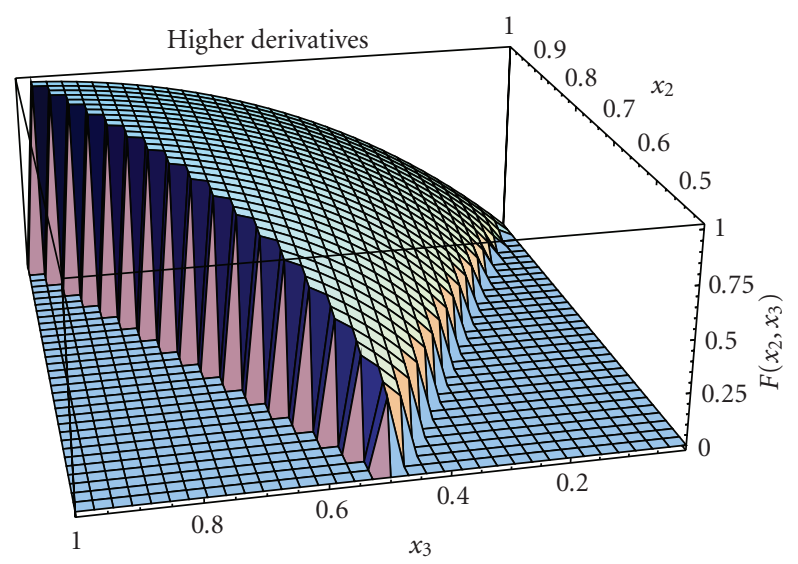

(a)

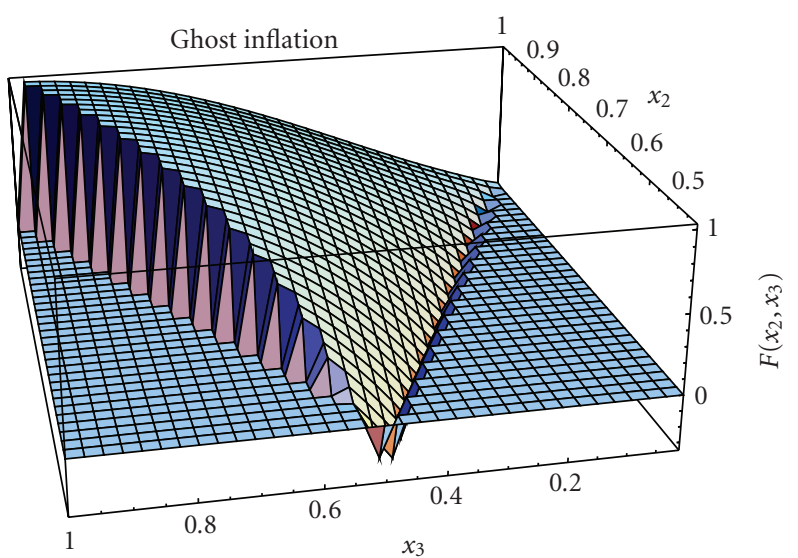

(b)

FIgURE 5: Plot of the function $F\left(1, x_{2}, x_{3}\right) x_{2}^{2} x_{3}^{2}$ for the inflation with higher derivatives as given by (22) (a) and the ghost inflation as given by (23) (b). The figures are normalized to have value 1 for equilateral configurations $x_{2}=x_{3}=1$ and set to zero outside the region $1-x_{2} \leq x_{3} \leq x_{2}$. Here $x_{3} \equiv k_{3} / k_{1}$ and $x_{2} \equiv k_{2} / k_{1}$. The figures are taken from Babich et al. 2003 [86].

where $\beta_{k_{j}}$ are the Bogoliubov coefficients which encode information about the initial conditions, $\eta_{0}$ is the initial conformal time and $\tilde{k}_{j}=\sum_{i} k_{i}-2 k_{j}$.

\section{The Cosmic Microwave Background Bispectrum}

Since the discovery of CMB by Penzias and Wilson in 1965 [117] and the first detection of CMB temperature anisotropies on large scales by the COBE DMR [118], the space satellite WMAP and over a dozens of balloon and ground-based experiments have characterized the $\mathrm{CMB}$ temperature anisotropies to a high accuracy and over a wide range of angular scales. The space satellite Planck which launched in 2009 will soon characterize the temperature anisotropies to even higher accuracy up to angular scales of $\ell_{\max } \approx 2500$. The CMB power spectrum is obtained by reducing all the information of $N_{\text {pix }}\left(\sim 10^{6}\right.$ for WMAP and $\sim 10^{7}$ for Planck). Such reduction is justified to obtain a fiducial model, given that the non-Gaussianities are expected to be small. With high quality data on the way, the field of non-Gaussianity is taking off. CMB bispectrum contains information which is not present in the power-spectrum and as we say in the previous section, is a unique probe of the early universe. 
The harmonic coefficients of the CMB anisotropy $a_{l m}=$ $T^{-1} \int d^{2} \hat{\mathbf{n}} \Delta T(\hat{\mathbf{n}}) Y_{\ell m}^{*}$ can be related to the primordial fluctuation $\Phi$ as

$$
a_{\ell m}^{p}=b_{\ell} 4 \pi(-i)^{\ell} \int \frac{d^{3} k}{(2 \pi)^{3}} \Phi(\mathbf{k}) g_{\ell}^{p}(k) Y_{\ell m}^{*}(\widehat{\mathbf{k}})+n_{\ell m},
$$

where $\Phi(\mathbf{k})$ is the primordial curvature perturbations, for a comoving wavevector $\mathbf{k}, g_{\ell}^{p}(r)$ is the radiation transfer function, where the index $p$ refers to either temperature $(T)$ or $E$-polarization $(E)$ of the CMB. A beam function $b_{\ell}$ and the harmonic coefficient of noise $n_{\ell m}$ are instrumental effects. Equation (26) is written for a flat background, but can easily be generalized.

Any non-Gaussianity present in the primordial perturbations $\Phi(\mathbf{k})$ gets transferred to the observed CMB via (26). The most common way to look for non-Gaussianity in the $\mathrm{CMB}$ is to study the bispectrum, the three-point function of temperature and polarization anisotropies in harmonic space. The CMB angular bispectrum is defined as

$$
B_{\ell_{1} \ell_{2} \ell_{3}, m_{1} m_{2} m_{3}}^{p q r} \equiv\left\langle a_{\ell_{1} m_{1}}^{p} a_{\ell_{2} m_{2}}^{q} a_{\ell_{3} m_{3}}^{r}\right\rangle
$$

and the angular-averaged bispectrum is

$$
B_{\ell_{1} \ell_{2} \ell_{3}}^{p q r}=\sum_{m_{1} m_{2} m_{3}}\left(\begin{array}{ccc}
\ell_{1} & \ell_{2} & \ell_{3} \\
m_{1} & m_{2} & m_{3}
\end{array}\right) B_{\ell_{1} \ell_{2} \ell_{3}, m_{1} m_{2} m_{3}}^{\mathrm{pqr}},
$$

where the matrix is the Wigner 3J symbol imposing selection rules which makes bispectrum zero unless

(i) $\ell_{1}+\ell_{2}+\ell_{3}=$ integer,

(ii) $m_{1}+m_{2}+m_{3}=0$,

(iii) $\left|\ell_{i}-\ell_{j}\right| \leq \ell_{k} \leq \ell_{i}+\ell_{j}$ for $i, j, k=1,2,3$.

Using (26) the bispectrum can be written as

$$
\begin{aligned}
B_{\ell_{1} \ell_{2} \ell_{3}}^{p q r}= & (4 \pi)^{3}(-i)^{\ell_{1}+\ell_{2}+\ell_{3}} \sum_{m_{1} m_{2} m_{3}}\left(\begin{array}{ccc}
\ell_{1} & \ell_{2} & \ell_{3} \\
m_{1} & m_{2} & m_{3}
\end{array}\right) \\
& \times \int \frac{d^{3} k_{1}}{(2 \pi)^{3}} \frac{d^{3} k_{2}}{(2 \pi)^{3}} \frac{d^{3} k_{3}}{(2 \pi)^{3}} Y_{\ell_{1} m_{1}}^{*}\left(\hat{\mathbf{k}}_{1}\right) Y_{\ell_{2} m_{2}}^{*}\left(\hat{\mathbf{k}}_{2}\right) \\
& \times Y_{\ell_{3} m_{3}}^{*}\left(\hat{\mathbf{k}}_{3}\right) g_{\ell_{1}}^{p}\left(k_{1}\right) g_{\ell_{2}}^{q}\left(k_{2}\right) g_{\ell_{3}}^{r}\left(k_{3}\right) \\
& \times\left\langle\Phi\left(\mathbf{k}_{1}\right) \Phi\left(\mathbf{k}_{2}\right) \Phi\left(\mathbf{k}_{3}\right)\right\rangle,
\end{aligned}
$$

where $\left\langle\Phi\left(\mathbf{k}_{1}\right) \Phi\left(\mathbf{k}_{2}\right) \Phi\left(\mathbf{k}_{3}\right)\right\rangle$ is the primordial curvature threepoint function as defined in (16).

To forecast constraints on non-Gaussianity using CMB data, we will perform a Fisher matrix analysis. The Fisher matrix for the parameters $p_{a}$ can be written as $[20,34,108]$

$$
\tilde{F}_{a b}=\sum_{\{i j k, p q r\}} \sum_{\ell_{1} \leq \ell_{2} \leq \ell_{3}} \frac{1}{\Delta \ell_{1} \ell_{2} \ell_{3}} \frac{\partial B_{\ell_{1} \ell_{2} \ell_{3}}^{p q r}}{\partial p_{a}}\left(\mathbf{C o v}^{-1}\right)_{i j k, p q r} \frac{\partial B_{\ell_{1} \ell_{2} \ell_{3}}^{i j k}}{\partial p_{b}} .
$$

The indices $a$ and $b$ run over all the parameters bispectrum depends on that we will assume all the cosmological parameters except $f_{\mathrm{NL}}$ to be known. Indices $i j k$ and pqr run over all the eight possible ordered combinations of temperature and polarization given by TTT, TTE, TET, ETT, TEE, ETE, EET and EEE, the combinatorial factor $\Delta_{\ell_{1} \ell_{2} \ell_{3}}$ equals 1 when all $\ell^{\prime}$ 's are different, 6 when $\ell_{1}=\ell_{2}=\ell_{3}$, and 2 otherwise. The covariance matrix Cov is obtained in terms of $C_{\ell}^{T T}, C_{\ell}^{E E}$, and $C_{\ell}^{T E}$ (see $[21,34]$ ) by applying Wick's theorem.

For non-Gaussianity of the local type, for which the functional form $F\left(k_{1}, k_{2}, k_{3}\right)$, is given by (18), we have

$$
\begin{aligned}
\frac{\partial B_{\ell_{1} \ell_{2} \ell_{3}}^{i j k k}}{\partial f_{\mathrm{NL}}}= & \sqrt{\frac{\left(2 \ell_{1}+1\right)\left(2 \ell_{2}+1\right)\left(2 \ell_{3}+1\right)}{4 \pi}}\left(\begin{array}{ccc}
\ell_{1} & \ell_{2} & \ell_{3} \\
0 & 0 & 0
\end{array}\right) \\
& \times 2 \int_{0}^{\infty} r^{2} d r\left[-\alpha_{\ell_{1}}^{i} \beta_{\ell_{2}}^{j} \beta_{\ell_{3}}^{k}+2 \text { perm. }\right]
\end{aligned}
$$

where the functions $\alpha$ and $\beta$ are given by

$$
\begin{gathered}
\alpha_{\ell}^{i}(r) \equiv \frac{2}{\pi} \int d k k^{2} g_{\ell}^{i}(k) j_{\ell}(k r), \\
\beta_{\ell}^{i}(r) \equiv \frac{2}{\pi} \int d k k^{-1} g_{\ell}^{i}(k) j_{\ell}(k r) \Delta_{\Phi} k^{n_{s}-1} .
\end{gathered}
$$

In the previous expression we use the dimensionless power spectrum amplitude $\Delta_{\Phi}$, which is defined by $P_{\Phi}(k)=$ $\Delta_{\Phi} k^{-3+\left(n_{s}-1\right)}$, where $n_{s}$ is the tilt of the primordial power spectrum. One can compute the transfer functions $g_{\ell}^{T}(k)$ and $g_{\ell}^{E}(k)$ using publicly available codes such as CMBfast [47] and CAMB [119]

In a similar way, from (21), one can derive the following expressions for the bispectrum derivatives in the equilateral case:

$$
\begin{gathered}
\frac{\partial B_{\ell_{1} \ell_{2} \ell_{3}}^{i j k}}{\partial f_{\mathrm{NL}}}=\sqrt{\frac{\left(2 \ell_{1}+1\right)\left(2 \ell_{2}+1\right)\left(2 \ell_{3}+1\right)}{4 \pi}}\left(\begin{array}{ccc}
\ell_{1} & \ell_{2} & \ell_{3} \\
0 & 0 & 0
\end{array}\right) \\
\times 6 \int r^{2} d r\left[-\alpha_{\ell_{1}}^{i} \beta_{\ell_{2}}^{j} \beta_{\ell_{3}}^{k}+2 \text { perm. }+\beta_{\ell_{1}}^{i} \gamma_{\ell_{2}}^{j} \delta_{\ell_{3}}^{k}\right. \\
\left.+5 \text { perm. }-2 \delta_{\ell_{1}}^{i} \delta_{\ell_{2}}^{j} \delta_{\ell_{3}}^{k}\right]
\end{gathered}
$$

where the functions $\delta$ and $\gamma$ are given by

$$
\begin{aligned}
& \gamma_{\ell}^{i}(r) \equiv \frac{2}{\pi} \int d k k g_{\ell}^{i}(k) j_{\ell}(k r) \Delta_{\Phi}^{1 / 3} k^{\left(n_{s}-1\right) / 3} \\
& \delta_{\ell}^{i}(r) \equiv \frac{2}{\pi} \int d k g_{\ell}^{T}(k) j_{\ell}(k r) \Delta_{\Phi}^{2 / 3} k^{2\left(n_{s}-1\right) / 3} .
\end{aligned}
$$


Recently, a new bispectrum template shape, an orthogonal shape, has been introduced [120] which characterizes the size of the signal $\left(f_{\mathrm{NL}}^{\text {ortho }}\right)$ which peaks both for equilateral and flat-triangle configurations. The shape of non-Gaussianities associated with $f_{\mathrm{NL}}^{\text {ortho }}$ is orthogonal to the one associated to $f_{\mathrm{NL}}^{\text {equil }}$. The bispectrum for orthogonal shape can be written as [120]

$$
\begin{gathered}
\frac{\partial B_{\ell_{1} \ell_{2} \ell_{3}}^{i j k}}{\partial f_{\mathrm{NL}}^{\text {ortho }}}=\sqrt{\frac{\left(2 \ell_{1}+1\right)\left(2 \ell_{2}+1\right)\left(2 \ell_{3}+1\right)}{4 \pi}}\left(\begin{array}{ccc}
\ell_{1} & \ell_{2} & \ell_{3} \\
0 & 0 & 0
\end{array}\right) \\
\quad \times 18 \int r^{2} d r\left[-\alpha_{\ell_{1}}^{i} \beta_{\ell_{2}}^{j} \beta_{\ell_{3}}^{k}+2 \text { perm. }+\beta_{\ell_{1}}^{i} \gamma_{\ell_{2}}^{j} \delta_{\ell_{3}}^{k}\right. \\
\left.+5 \text { perm. }-\frac{2}{3} \delta_{\ell_{1}}^{i} \delta_{\ell_{2}}^{j} \delta_{\ell_{3}}^{k}\right] .
\end{gathered}
$$

4.1. Estimator. An unbiased bispectrum-based minimum variance estimator for the nonlinearity parameter in the limit of full sky and homogeneous noise can be written as $[17,20,25]$

$$
\hat{f}_{\mathrm{NL}}=\frac{1}{N} \sum_{\ell_{i} m_{i}}\left(\begin{array}{ccc}
\ell_{1} & \ell_{2} & \ell_{3} \\
m_{1} & m_{2} & m_{3}
\end{array}\right) \frac{B_{\ell_{1} \ell_{2} \ell_{3}}}{C_{\ell_{1}} C_{\ell_{2}} C_{\ell_{3}}} a_{\ell_{1} m_{1}} a_{\ell_{2} m_{2}} a_{\ell_{3} m_{3}},
$$

where $B_{\ell_{1} \ell_{2} \ell_{3}}$ is angle averaged theoretical CMB bispectrum for the model in consideration. The normalization $N$ can be calculated to require the estimator to be unbiased, $\left\langle\hat{f}_{\mathrm{NL}}\right\rangle=$ $f_{\mathrm{NL}}$. If the bispectrum $B_{\ell_{1} \ell_{2} \ell_{3}}$ is calculated for $f_{\mathrm{NL}}=1$ then the normalization takes the following form:

$$
N=\sum_{\ell_{i}} \frac{\left(B_{\ell_{1} \ell_{2} \ell_{3}}\right)^{2}}{C_{\ell_{1}} C_{\ell_{2}} C_{\ell_{3}}}
$$

The estimator for non-Gaussianity, (36), can be simplified using (26) to yield

$$
\begin{aligned}
\hat{f}_{\mathrm{NL}}= & \frac{1}{N} \cdot \sum_{l_{i} m_{i}} \int d^{2} \hat{n} Y_{l_{1} m_{1}}(\hat{n}) Y_{l_{2} m_{2}}(\hat{n}) Y_{l_{3} m_{3}}(\hat{n}) \\
& \times \int_{0}^{\infty} r^{2} d r j_{l_{1}}\left(k_{1} r\right) j_{l_{2}}\left(k_{2} r\right) j_{l_{3}}\left(k_{3} r\right) C_{l_{1}}^{-1} C_{l_{2}}^{-1} C_{l_{3}}^{-1} \\
& \times \int \frac{2 k_{1}^{2} d k_{1}}{\pi} \frac{2 k_{2}^{2} d k_{2}}{\pi} \frac{2 k_{3}^{2} d k_{3}}{\pi} F\left(k_{1}, k_{2}, k_{3}\right) \Delta_{l_{1}}^{T}\left(k_{1}\right) \\
& \times \Delta_{l_{2}}^{T}\left(k_{2}\right) \Delta_{l_{3}}^{T}\left(k_{3}\right) a_{l_{1} m_{1}} a_{l_{2} m_{2}} a_{l_{3} m_{3}},
\end{aligned}
$$

where $F\left(k_{1}, k_{2}, k_{3}\right)$ is a shape of 3-point function as defined in (16). Given the shape $F\left(k_{1}, k_{2}, k_{3}\right)$, one is interested in, it is conceptually straightforwardto constrain the non- linearity parameter from the CMB data. Unfortunately the computation time for the estimate scales as $N_{\text {pix }}^{5 / 2}$, which is computationally challenging as even for the WMAP data the number of pixels, is of order $N_{\text {pix }} \sim 10^{6}$. The scaling can be understood by noting that each spherical harmonic transform scales as $N_{\text {pix }}^{3 / 2}$ and the estimator requires $\ell^{2}(\propto$ $N_{\text {pix }}$ ) number of spherical harmonic transforms.

The computational cost decreases if the shape can be factorized as

$$
F\left(k_{1}, k_{2}, k_{3}\right)=f_{1}\left(k_{1}\right) f_{2}\left(k_{2}\right) f_{3}\left(k_{3}\right)
$$

with which the estimator simplifies to

$$
\begin{aligned}
\hat{f}_{\mathrm{NL}}= & \frac{1}{N} \cdot \int_{0}^{\infty} d^{2} \hat{n} \int_{0}^{\infty} r^{2} d r \prod_{i=1}^{3} \sum_{l_{i} m_{i}} \\
& \times \int \frac{2 k^{2} d k}{\pi} j_{l_{i}}(k r) f_{i}(k) \Delta_{l_{i}}^{T}(k) C_{l_{i}}^{-1} a_{l_{i} m_{i}} Y_{l_{i} m_{i}}(\hat{n}),
\end{aligned}
$$

and computational cost now scales as $N_{\text {pix }}^{3 / 2}$. For Planck $\left(N_{\text {pix }} \sim 5 \times 10^{7}\right)$ this translates into a speed-up by factors of millions, reducing the required computing time from thousands of years to just hours and thus making $f_{\mathrm{NL}}$ estimation feasible for future surveys. The speed of the estimator now allows sufficient number of Monte Carlo simulations, to characterize its statistical properties in the presence of real world issues such as instrumental effects, partial sky coverage, and foreground contamination. Using the Monte Carlo simulations it has been shown that estimator is indeed optimal, where optimality is defined by saturation of the Cramer Rao bound, if noise is homogeneous. Note that even for the nonfactorizable shapes, by using the flat sky approximation and interpolating between the modes, one can estimating $f_{\mathrm{NL}}$ in a computationally efficient way [121].

The extension of the estimator of $f_{\mathrm{NL}}$ from the temperature data [17] to include both the temperature and polarization data of the CMB is discussed in Babich and Zaldarriaga [34] and Yadav et al. [20, 21, 35]. Summarizing briefly, we construct a cubic statistic as a combination of (appropriately filtered) temperature and polarization maps which is specifically sensitive to the primordial perturbations. This is done by reconstructing a map of primordial perturbations and using that to define a fast estimator. We also show that this fast estimator is equivalent to the optimal estimator by demonstrating that the inverse of the covariance matrix for the optimal estimator [34] is the same as the product of inverses we get in the fast estimator. The estimator still takes only $N_{\text {pix }}^{3 / 2}$ operations in comparison to the full bispectrum calculation which takes $N_{\text {pix }}^{5 / 2}$ operations.

For a given shape, the estimator for non-linearity parameter can be written as $\hat{f}_{\mathrm{NL}}=\hat{S}_{\text {shape }} / N_{\text {shape, }}$ where for the 
equilateral, local and orthogonal shapes, the $S_{\text {shape }}$ can be written as

$$
\begin{aligned}
\hat{S}_{\text {equilateral }}= & \frac{3}{f_{\text {sky }}} \int r^{2} d r \int d^{2} \hat{n} \\
& \times\left[B(\hat{n}, r) B(\hat{n}, r) A(\hat{n}, r)+\frac{2}{3} D(\hat{n}, r)^{3}\right. \\
& \quad-2 B(\hat{n}, r) C(\hat{n}, r) D(\hat{n}, r)], \\
\hat{S}_{\text {local }}= & \frac{1}{f_{\text {sky }}} \int r^{2} d r \int d^{2} \hat{n} B(\hat{n}, r) B(\hat{n}, r) A(\hat{n}, r), \\
\hat{S}_{\text {orthogonal }}= & \frac{9}{f_{\text {sky }}} \int r^{2} d r \int d^{2} \hat{n} \\
& \times\left[B(\hat{n}, r) B(\hat{n}, r) A(\hat{n}, r)+\frac{8}{9} D(\hat{n}, r)^{3}\right. \\
& \quad-2 B(\hat{n}, r) C(\hat{n}, r) D(\hat{n}, r)],
\end{aligned}
$$

with

$$
\begin{aligned}
& B(\hat{n}, r) \equiv \sum_{i p} \sum_{l m}\left(C^{-1}\right)^{i p} a_{\ell m}^{i} \beta_{\ell}^{p}(r) Y_{\ell m}(\hat{n}), \\
& C(\hat{n}, r) \equiv \sum_{i p} \sum_{l m}\left(C^{-1}\right)^{i p} a_{\ell m}^{i} \beta_{\ell}^{p}(r) Y_{\ell m}(\hat{n}), \\
& A(\hat{n}, r) \equiv \sum_{i p} \sum_{l m}\left(C^{-1}\right)^{i p} a_{\ell m}^{i} \alpha_{\ell}^{p}(r) Y_{\ell m}(\hat{n}), \\
& D(\hat{n}, r) \equiv \sum_{i p} \sum_{l m}\left(C^{-1}\right)^{i p} a_{\ell m}^{i} \beta_{\ell}^{p}(r) Y_{\ell m}(\hat{n}),
\end{aligned}
$$

and $f_{\text {sky }}$ is a fraction of sky. Index $i$ and $p$ can either be $T$ or E :

$$
\begin{aligned}
N= & \sum_{i j k p q r 2 \leq \ell_{1} \leq \ell_{2} \leq \ell_{3}} \frac{1}{\Delta \ell_{1} \ell_{2} \ell_{3}} \\
& \times B_{\ell_{1} \ell_{2} \ell_{3}}^{p q r \text { prim }}\left(C^{-1}\right)_{\ell_{1}}^{i p}\left(C^{-1}\right)_{\ell_{2}}^{j q}\left(C^{-1}\right)_{\ell_{3}}^{k r} B_{\ell_{1} \ell_{2} \ell_{3}}^{i j k, \text { prim }} .
\end{aligned}
$$

Indices $i, j, k, p, q$ and $r$ can either be $T$ or $E$. Here, $\Delta_{\ell_{1} \ell_{2} \ell_{3}}$ is 1 when $\ell_{1} \neq \ell_{2} \neq \ell_{3}, 6$ when $\ell_{1}=\ell_{2}=\ell_{3}$, and 2 otherwise: $B_{\ell_{1} \ell_{2} \ell_{3}}^{p q r, p r i m}$ is the theoretical bispectrum for $f_{\mathrm{NL}}=1$ [21].

It has been shown that the previous estimators defined in (42) are minimum variance amongst bispectrum-based estimators for full sky coverage and homogeneous noise [21]. To be able to deal with the realistic data, the estimator has to be able to deal with the inhomogeneous noise and foreground masks. The estimator can be generalized to deal with partial sky coverage as well as inhomogeneous noise by adding a linear term to $\hat{S}_{\text {prim }}: \hat{S}_{\text {prim }} \rightarrow \widehat{S}_{\text {prim }}+\hat{S}_{\text {prim }}^{\text {linear }}$. For the temperature only case, this has been done in [25]. Following the same argument, we find that the linear term for the combined analysis of CMB temperature and polarization data is given by

$$
\begin{aligned}
& \hat{S}_{\text {prim }}^{\text {linear }}=\frac{-1}{f_{\text {sky }}} \int r^{2} d r \int d^{2} \hat{n}\left\{2 B(\hat{n}, r)\left\langle A_{\text {sim }}(\hat{n}, r) B_{\text {sim }}(\hat{n}, r)\right\rangle_{\mathrm{MC}}\right. \\
& \left.+A(\hat{n}, r)\left\langle B_{\mathrm{sim}}^{2}(\hat{n}, r)\right\rangle_{\mathrm{MC}}\right\},
\end{aligned}
$$

where $A_{\text {sim }}(\hat{n}, r)$ and $B_{\text {sim }}(\hat{n}, r)$ are the $A$ and $B$ maps generated from Monte Carlo simulations that contain signal and noise, and $\langle\cdots\rangle$ denotes the average over the Monte Carlo simulations.

The generalized estimator is given by

$$
\hat{f}_{\mathrm{NL}}=\frac{\hat{S}_{\text {prim }}+\hat{S}_{\text {prim }}^{\text {linear }}}{N} .
$$

Note that $\left\langle\hat{S}_{\text {prim }}^{\text {linear }}\right\rangle_{\mathrm{MC}}=-\left\langle\widehat{S}_{\text {prim }}\right\rangle_{\mathrm{MC}}$, and this relation also holds for the equilateral shape. Therefore, it is straightforward to find the generalized estimator for the equilateral shape: first, find the cubic estimator of the equilateral shape, $\hat{S}_{\text {equil. }}$, and take the Monte Carlo average, $\left\langle\hat{S}_{\text {equil. }}\right\rangle_{\text {MC }}$. Let us suppose that $\hat{S}_{\text {equil. }}$ contains terms in the form of $A B C$, where $A, B$, and $C$ are some filtered maps. Use the Wick's theorem to rewrite the average of a cubic product as $\langle A B C\rangle_{\mathrm{MC}}=\langle A\rangle_{\mathrm{MC}}\langle B C\rangle_{\mathrm{MC}}+\langle B\rangle_{\mathrm{MC}}\langle A C\rangle_{\mathrm{MC}}+$ $\langle C\rangle_{\mathrm{MC}}\langle A B\rangle_{\mathrm{MC}}$. Finally, remove the MC average from single maps and replace maps in the product with the simulated maps $\langle A\rangle_{\mathrm{MC}}\langle B C\rangle_{\mathrm{MC}}+\langle B\rangle_{\mathrm{MC}}\langle A C\rangle_{\mathrm{MC}}+\langle C\rangle_{\mathrm{MC}}\langle A B\rangle_{\mathrm{MC}} \rightarrow$ $A\left\langle B_{\text {sim }} C_{\text {sim }}\right\rangle_{\mathrm{MC}}+B\left\langle A_{\text {sim }} C_{\text {sim }}\right\rangle_{\mathrm{MC}}+C\left\langle A_{\text {sim }} B_{\text {sim }}\right\rangle_{\mathrm{MC}}$. This operation gives the correct expression for the linear term, both for the local form and the equilateral form.

The main contribution to the linear term comes from the inhomogeneous noise and sky cut. For the temperature only case, most of the contribution to the linear term comes from the inhomogeneous noise, and the partial sky coverage does not contribute much to the linear term. This is because the sky-cut induces a monopole contribution outside the mask. In the analysis, one subtracts the monopole from outside the mask before measuring $\hat{S}_{\text {prim }}$, which makes the linear contribution from the mask small [25]. For a combined analysis of the temperature and polarization maps, however, the linear term does get a significant contribution from a partial sky coverage. Subtraction of the monopole outside of the mask is of no help for polarization, as the monopole does not exist in the polarization maps by definition. (The lowest relevant multipole for polarization is $l=2$.)

The estimator is still computationally efficient, taking only $N_{\text {pix }}^{3 / 2}$ (times the $r$ sampling, which is of order 100) operations in comparison to the full bispectrum calculation which takes $N_{\text {pix }}^{5 / 2}$ operations. Here $N_{\text {pix }}$ refers to the total number of pixels. For Planck, $N_{\text {pix }} \sim 5 \times 10^{7}$, and so the full bispectrum analysis is not feasible while our analysis is.

\section{Constraints from the CMB Bispectrum}

5.1. Current Status. Currently the the Wilkinson Microwave Anisotropy Probe (WMAP) satellite provides the "best" 
(largest number of signal dominated modes) CMB data for non-Gaussianity analysis. Over the course of WMAP operation the field of non-Gaussianity has made vast progress both in terms of theoretical predictions of non-Gaussianities from inflation and improvement in the bispectrum- based estimators. At the time of WMAP's first data release in 2003 the estimator was suboptimal in the presence of partial sky coverage and/or inhomogeneous noise. With the sub-optimal estimator, one could not use the entirety of WMAP data and only the data up to $l_{\max } 350$ were used to obtain the constraint $f_{\mathrm{NL}}^{\text {local }}=38 \pm 96(2 \sigma)[23]$. These limits were around 30 times better than the previous constraints of $\left|f_{\mathrm{NL}}\right|<1.5 \times 10^{3}$ from the Cosmic Background Explorer (COBE) satellite [22].

By the time of second WMAP release in 2007, the estimator was generalized by adding a linear to the KSW estimator which allows to deal with partial sky coverage and inhomogeneous noise. The idea of adding a linear term to reduce excess variance due to noise inhomogeneity was introduced in [25]. Applied to a combination of the Q, V, and $\mathrm{W}$ channels of the WMAP 3 -year data up to $l_{\max } \sim 400$, this estimator had yielded the tightest constraint at the time on $f_{\mathrm{NL}}$ as $-36<f_{\mathrm{NL}}<100(2 \sigma)$ [26]. This estimator was further generalized to utilize both the temperature and $E$ polarization information in [20], where it was pointed out that the linear term had been incorrectly implemented in 30 of [25]. Using MonteCarlo simulations it has been shown that this corrected estimator is nearly optimal and enables analysis of the entire WMAP data without suffering from a blow-up in the variance at high multipoles ${ }^{6}$. The first analysis using this estimator shows an evidence of non-Gaussianity of local type at around $2.8 \sigma$ in the WMAP 3-year data. Independent analysis shows the evidence of non-Gaussianity at lower significance, around $2.5 \sigma$ (see Table 1 ).

By the time of the third WMAP data release (with 5-year obsevational data) in 2008 the $f_{\mathrm{NL}}$ estimation technique was improved further by implementing the covariance matrix including inhomogeneoous noise to make the estimator completely optimal [122]. Using the optimal estimator and using the entirety of WMAP 3-year data, there is an evidence for non-Gaussianity of local type at around $2.5 \sigma$ level $f_{\mathrm{NL}} \approx$ $58 \pm 23(1 \sigma)[122]$. However with WMAP 5-year data the significance goes down from $\sim 2.5 \sigma$ to $\sim 1.8 \sigma$ [122]. Table 2 compares the constraints obtained by different groups using WMAP 3-year and WMAP 5-year data. Figure 6 shows this comparison in more detail, showing the constraints also as a function of maximum multipole $l_{\max }$ used in the analysis. Few comments are in place: (1) constraints on $f_{\mathrm{NL}}$ from WMAP 3-year data as a function of $l_{\max }$ show a trend where the mean value rises at around $l_{\max }=450$, below which data is consistent with Gaussianity and above which there is deviation from Gaussianity at above $2 \sigma$. The result becomes roughly independent of $\ell_{\max }>550$ with evidence for nonGaussianity at around $2.5 \sigma$ level (2) independent analysis and using different estimators (optimal and near-optimal with linear term) see this deviation from non-Gaussianity at around 2.5 $\sigma$ in WMAP 3-year data, (3) significance of nonGaussianity goes down to around $2 \sigma$ with WMAP 5-year data. The drop in the mean value between WMAP 3-year and 5 -year data can be attributed to statistical shift.
The best constraints on the equilateral and orthogonal shape of non-Gaussianity using the WMAP 5-year data are $f_{\mathrm{NL}}^{\text {equil }}=155 \pm 140(1 \sigma)$ and $f_{\mathrm{NL}}^{\text {orthog. }}=149 \pm 110(1 \sigma)$ respectively [120].

As we were completing this paper, the WMAP 7-year data was released, with constraints $f_{\mathrm{NL}}^{\text {local }}=32 \pm 21(1 \sigma), f_{\mathrm{NL}}^{\text {equil. }}=$ $26 \pm 140(1 \sigma)$, and $f_{\mathrm{NL}}^{\text {orthog }}=-202 \pm 104(1 \sigma)[123]$.

5.2. Future Prospects. Now we discuss the future prospects of using the bispectrum estimators for constraining the nonlinearity parameter $f_{\mathrm{NL}}$ for local and equilateral shapes. We compute the Fisher bounds for three experimental setups, (1) cosmic variance limited experiment with perfect beam (ideal experiment hereafter), (2) Planck satellite with and noise sensitivity $\Delta_{p}=56 \mu \mathrm{K}$-arcmin and beam FWHM $\sigma=7^{\prime}$, and (3) a futuristic CMBPol-like satellite experiment with noise sensitivity $\Delta_{p}=1.4 \mu \mathrm{K}$-arcmin and beam FWHM $\sigma=4^{\prime}$ (CMBPol hereafter). Beside $f_{\mathrm{NL}}$ we fix all the other cosmological parameters to a standard fiducial model with a flat $\Lambda C D M$ cosmology, with parameters described by the best fit to WMAP 5-year results [48], given by $\Omega_{b}=0.045, \Omega_{c}=0.23, H_{0}=70.5, n_{s}=0.96, n_{t}=0.0$, and $\tau=0.08$. We calculate the theoretical CMB transfer functions and power spectrum from publicly available code CMBFAST [47]. We also neglect any non-Gaussianity which can be generated during recombination or there after. We discuss the importance and effect of these nonprimordial non-Gaussianities in the next section.

The scaling of signal-to-noise as a maximum multipole $l_{\max }$ for the local $[124,125]$ and equilateral model [126] are

$$
\left(\frac{S}{N}\right)_{\text {local }} \propto \ln \ell_{\max }, \quad\left(\frac{S}{N}\right)_{\text {equil }} \propto \sqrt{\ell_{\max }} .
$$

In principle one could go to arbitrary high $l_{\max }$ but in reality secondary signals will certainly overwhelm primary signal beyond $l_{\max }>3000$ : we restrict to the analysis to $l_{\max }=3000$. In Figure 7 , we show the $1 \sigma$ Fisher bound as function of maximum multipole $\ell_{\max }$, for local and equilateral type of non-Gaussianity. For both local and equilateral cases, we show the Fisher bound for the analysis using only the CMB temperature information (TTT), only the CMB polarization information (EEE), and the combined temperature and polarization analysis. Note that by having both the temperature and E-polarization information one can improve the sensitivity by combining the information. Apart from combining the $T$ and $E$ signal, one can also do cross-checks and diagnostics by independently analysing the data. Temperature and polarization will have different foregrounds and instrumental systematics.

A CMBPol-like experiment will be able to achieve the sensitivity of $\Delta f_{\mathrm{NL}}^{\text {local }} \simeq 2(1 \sigma)$ for non-Gaussianity of local type and $\Delta f_{\mathrm{NL}}^{\text {equil. }} \simeq 13(1 \sigma)$ for non-Gaussianity of equilateral type. For the local type of non-Gaussianity, this amounts to an improvement of about a factor of 2 over the Planck satellite and about a factor of 12 over current best constraints. These estimates assume that foreground cleaning can be done perfectly that is, the effect of residual foregrounds has 
TABLE 1: Hint of local non-Gaussianity at $2 \sigma$ level.

\begin{tabular}{lccc}
\hline Data (mask, estimator) & $f_{\text {NL }}^{\text {local }} \pm 1 \sigma$ error & Deviation from Gaussianity & \\
\hline WMAP 3-year (Kp0, near-optimal) & $87 \pm 31$ & $2.8 \sigma$ & Yadav and Wandelt [28] \\
WMAP 3-year (KQ75, optimal) & $58 \pm 23$ & $2.5 \sigma$ & Smith et al. [122] \\
WMAP 3-year (Kp0, near-optimal) & $69 \pm 30$ & $2.3 \sigma$ & Smith et al. [122] \\
WMAP 5-year (KQ75, near-optimal) & $51 \pm 30$ & $1.7 \sigma$ & Komatsu et al. [48] \\
WMAP 5-year (KQ75, optimal) & $38 \pm 21$ & $1.8 \sigma$ & Smith et al. [122] \\
\hline
\end{tabular}

The difference between the results by $[28,122]$ for WMAP 3-year data using the Kp0 mask can be a result of different choices of weighting in the near-optimal estimator. The optimal estimator has a unique weighting scheme.

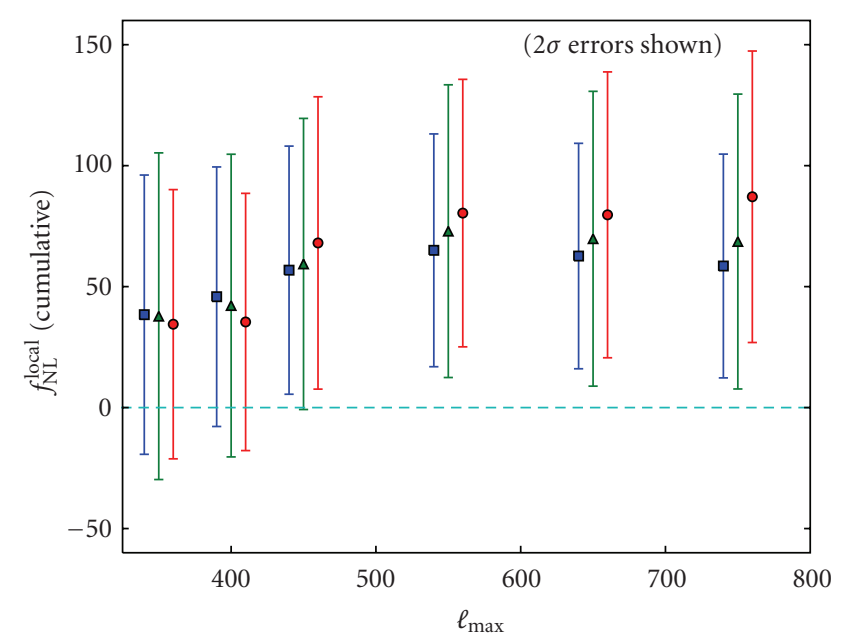

- 3-year, optimal foreground marginalized [122]

А 3-year, near-optimal raw-map [122]

- 3-year, near-optimal raw-map from [28]

(a)

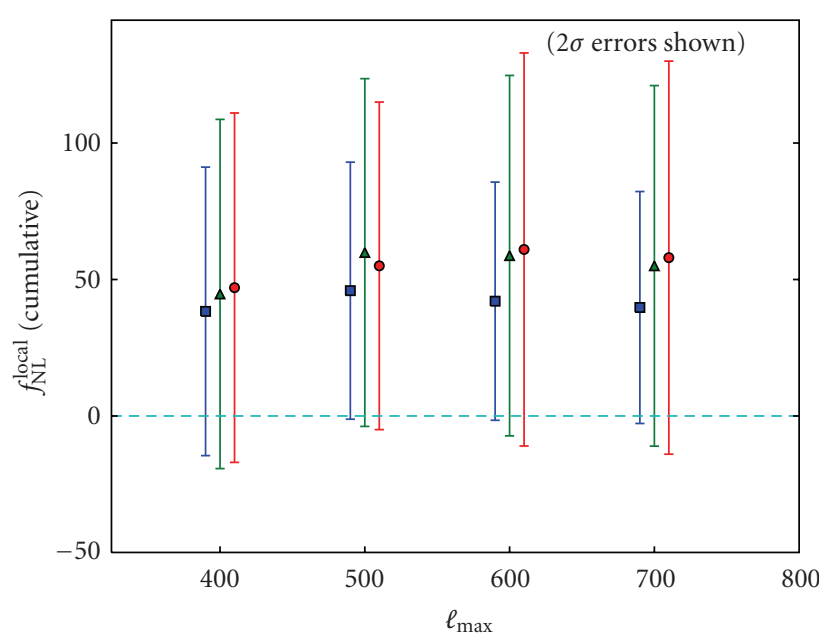

- 5-year, optimal foreground marginalized [122]

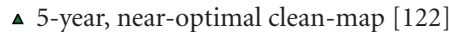

- 5-year, near-optimal clean-map from [48]

(b)

FIGURE 6: (a) Constraints on local $f_{\mathrm{NL}}$ using WMAP 3-year data as a function of maximum multipole $\ell_{\max }$ used in the analysis. The red circles are the results obtained using near optimal estimator by Yadav and Wandelt [28]. The green triangles are using the the near-optimal estimator by Smith et al. [122]. The blue square results are obtained using either the optimal estimator by Smith et al. [122]. For all the three analysis Kp0 mask was used. (b) Comparison between the 5-year results (optimal estimator, raw maps) reported in Komatsu et al. [48] and results obtained using the optimal or suboptimal estimator by Smith et al. [122].

been neglected. Also the contribution from unresolved point sources and secondary anisotropies such as ISW-lensing and SZ-lensing has been ignored.

Running Non-Gaussianity. The primordial non-Gaussian parameter $f_{\mathrm{NL}}$ has been shown to be scaledependent in several models of inflation with a variable speed of sound, such as Dirac-Born-Infeld (DBI) models. Starting from a simple ansatz for a scale-dependent amplitude of the primordial curvature bispectrum for primordial non-Gaussianity,

$$
f_{\mathrm{NL}} \longrightarrow f_{\mathrm{NL}}\left(\frac{K}{k_{p}}\right)^{n_{\mathrm{NG}}}
$$

where $K \equiv\left(k_{1} k_{2} k_{3}\right)^{1 / 3}$ and $k_{p}$ is a pivot point. The primordial bispectrum is therefore determined in terms of two parameters: the amplitude $f_{\mathrm{NL}}$ and the new parameter $n_{\mathrm{NG}}$ quantifying its running. One can generalize the Fisher matrix analysis of the bispectra of the temperature and polarization of the $\mathrm{CMB}$ radiation and derive the expected constraints on the parameter $n_{\mathrm{NG}}$ that quantifies the running of $f_{\mathrm{NL}}(k)$ for current and future CMB missions such as WMAP, Planck, and CMBPol. We will consider some nonzero $f_{\mathrm{NL}}$ as our fiducial value for the Fisher matrix evaluation. Clearly, in order to be able to constrain a scaledependence of $f_{\mathrm{NL}}$, its amplitude must be large enough to produce a detection. If $f_{\mathrm{NL}}$ is too small to be detected ( $f_{\mathrm{NL}}<2$ is a lowest theoretical limit even for the ideal experiment), we will obviously not be able to measure any of its features, either. The following we will then always consider a fiducial value of $f_{\mathrm{NL}}$ large enough to enable a detection. Figure 8 shows the $1-\sigma$ joint constraints on $f_{\mathrm{NL}}$ and $n_{\mathrm{NG}}$. In the event of a significant detection of the non-Gaussian component, corresponding to $f_{\mathrm{NL}}=50$ for the local model and 


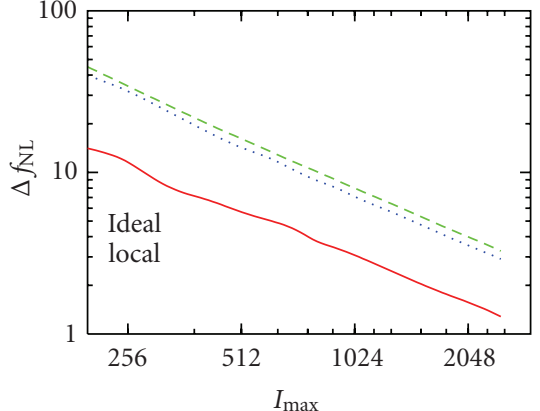

(a)

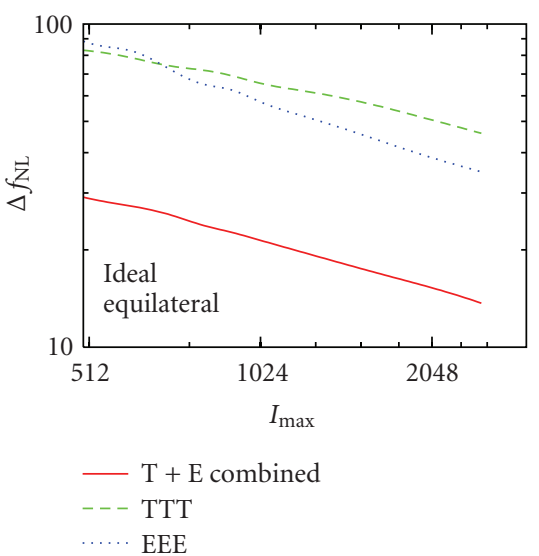

(d)

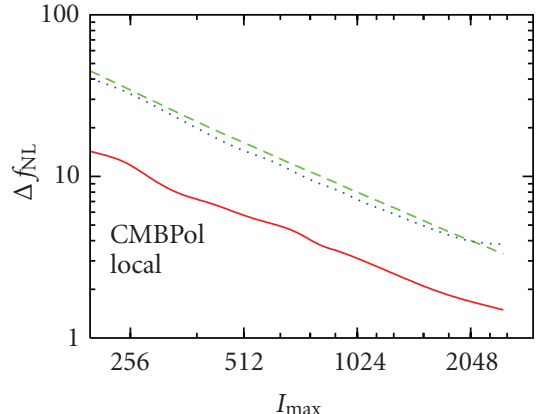

(b)

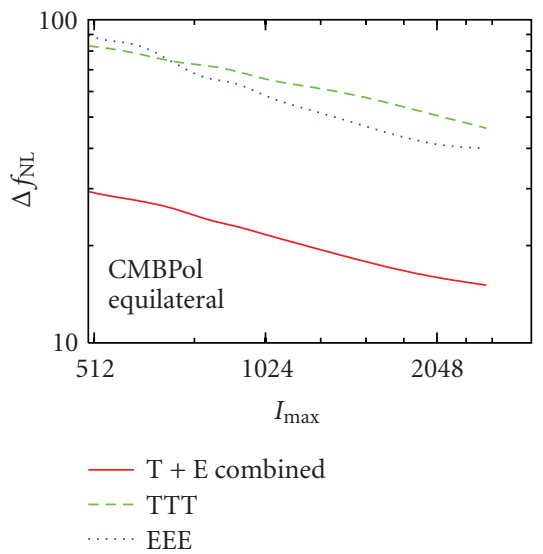

(e)

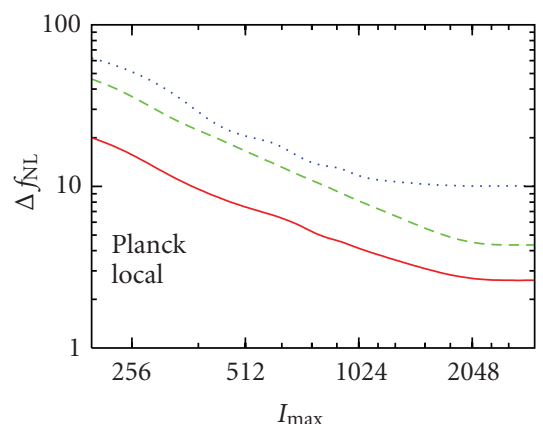

(c)

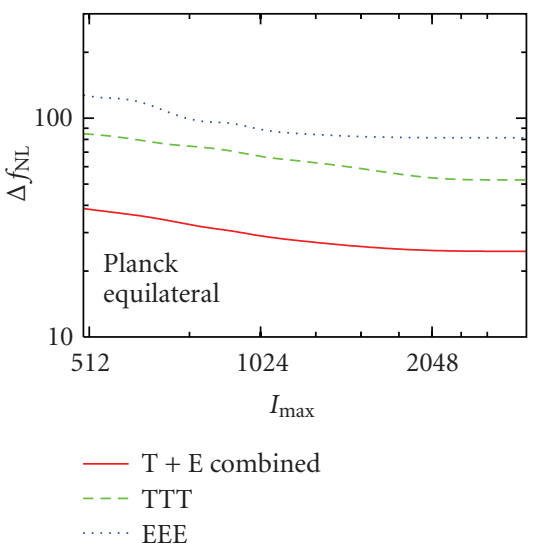

(f)

FIGURE 7: Fisher predictions for minimum detectable $f_{\mathrm{NL}}$ (at $1 \sigma$ ) as a function of maximum multipole $\ell_{\max }$. Upper panels are for the local model while ((d),(e), and (f)) are for the equilateral model. ((a) and (d)) show an ideal experiment, ((b) and(e)) are for CMBPol like experiment with noise sensitivity $\Delta_{p}=1.4 \mu \mathrm{K}$-arcmin and beam FWHM $\sigma=4^{\prime}$ and ((c) and (f)) are for Planck- like satellite with and noise sensitivity $\Delta_{p}=40 \mu \mathrm{K}$-arcmin and beam FWHM $\sigma=5^{\prime}$. In all the panels, the solid lines represent temperature and polarization combined analysis; dashed lines represent temperature only analysis; dot-dashed lines represent polarization only analysis.

$f_{\mathrm{NL}}=100$ for the equilateral model of non-Gaussianity, is able to determine $n_{\mathrm{NG}}$ with a $1-\sigma$ uncertainty of $\Delta n_{\mathrm{NG}} \simeq 0.1$ and $\Delta n_{\mathrm{NG}} \simeq 0.3$, respectively, for the Planck mission and a factor of two better for CMBPol. In addition to CMB, one can include the information of the galaxy power spectrum, galaxy bispectrum, and cluster number counts as a probe of nonGaussianity on small scales to further constrain the two parameters [127].

5.3. Contaminations. A detection of non-Gaussianity has profound implications on our understanding of the early Universe. Hence it is important to know and quantify all the possible sources of non-Gaussianities in the CMB. Here we highlight some sources of non-Gaussianities due to second-order anisotropies after last scattering surface and during recombination. The fact that Gaussian initial conditions imply Gaussianity of the $\mathrm{CMB}$ is only true at linear order. We will also discuss the effects of instrumental effects and uncertainties in the cosmological parameters on the bispectrum estimate.

5.3.1. Secondary Non-Gaussianities. Current analysis of the $\mathrm{CMB}$ data ignore the contributions from the secondary
non-Gaussianities. For WMAP resolutions it may not be a bad approximation. Studies of the dominant secondary anisotropies conclude that they are negligible for the analysis of the WMAP data for $l_{\max }<800[108,128]$. However on smaller angular scales several effects start to kick in, for example, (1) the bispectrum contribution due to unresolved point source like thermal Sunyaev-Zeldovich clusters or standard radio sources, (2) three-way correlations between primary $\mathrm{CMB}$, lensed $\mathrm{CMB}$, and secondary anisotropies. We will refer to the bispectrum generated due to these threeway correlations as $B^{\text {secondary- } \kappa}$, where some secondaries are, the integrated Sachs-Wolfe (ISW) $B^{\text {ISW- }}$, Sunyaev-Zeldovich signal and Rees-Sciama $[23,108,128-131]$.

For Future experiments such as Planck and CMBPOl the joint estimation of primordial and secondary bispectrum will be required. The observed bispectrum in general would take the following form:

$$
\begin{aligned}
\hat{B}_{\ell_{1} \ell_{2} \ell_{3}}^{\mathrm{obs}}= & f_{\mathrm{NL}} B_{\ell_{1} \ell_{2} \ell_{3}}^{\mathrm{prim}}+b_{\mathrm{ps}} B_{\ell_{1} \ell_{2} \ell_{3}}^{\mathrm{ps}}+A_{S Z} B_{\ell_{1} e_{2} \ell_{3}}^{\mathrm{SZ}-\kappa} \\
& +A_{\mathrm{ISW}} B_{\ell_{1} \ell_{2} \ell_{3}}^{\mathrm{ISW}-\kappa}+\cdots .
\end{aligned}
$$

The amplitude of bispectrum due to primary-lensingsecondary cross-correlation is proportional to the product 


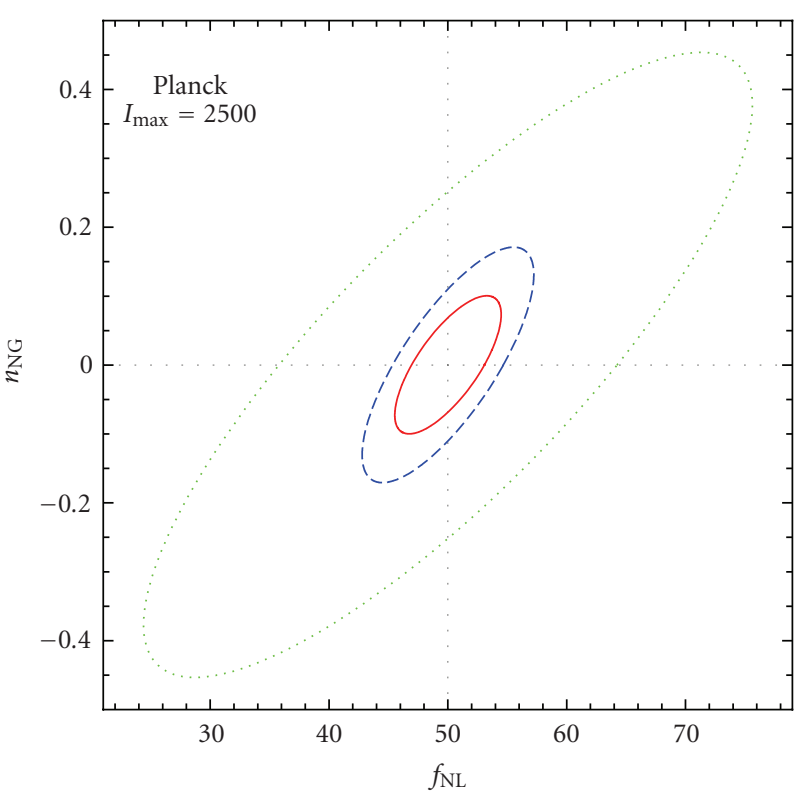

(a)

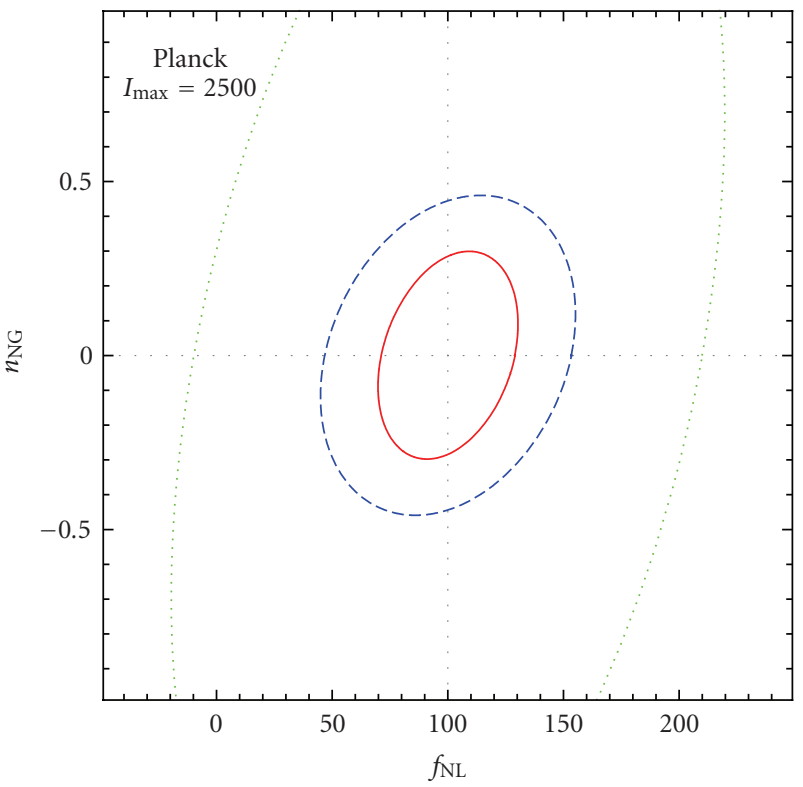

(c)

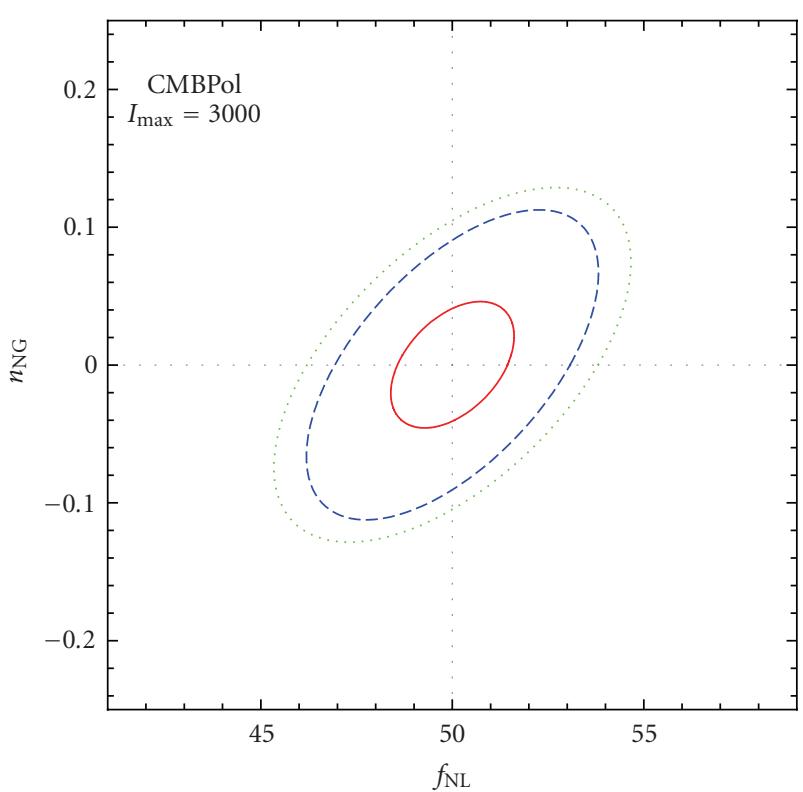

(b)

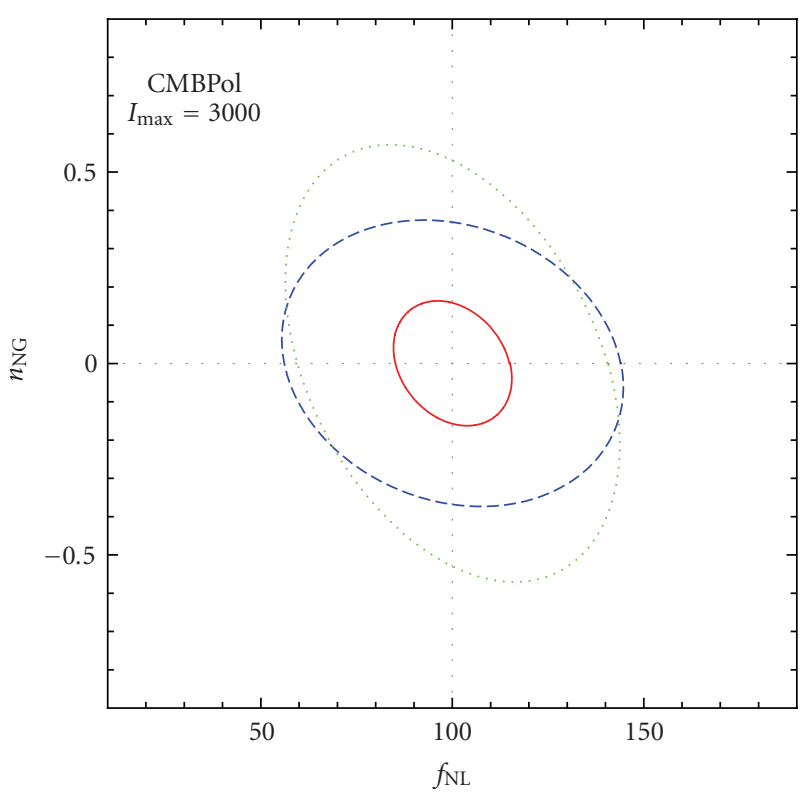

(d)

FIGURE 8: $1-\sigma$ constraints on $f_{\mathrm{NL}}$ and $n_{\mathrm{NG}}$ for local (a) and (b) and equilateral model (c) and (d) assuming $k_{p}=0.04 \mathrm{Mpc}^{-1}$ and fiducial values $f_{\mathrm{NL}}=50, n_{\mathrm{NG}}=0$ for the local case and $f_{\mathrm{NL}}=100, n_{\mathrm{NG}}=0$ for the equilateral case. Dashed lines correspond to the limits from the temperature information alone, dotted lines to polarization $(E E E)$, while the continuous lines correspond to all bispectrum combinations. We consider Planck (a) and (c) and CMBPOl-like (b) and (d) CMB experiment.

of primary CMB power-spectrum and power spectrum of cross-correlation between secondary and lensing signals.

The reduced bispectrum from the residual point sources (assuming Poisson distributed) is constant, that is, $b_{\ell_{1} \ell_{2} \ell_{3}}^{\mathrm{ps}}=$ constant. The value of the constant will depend on the flux limit at which the point source can be detected and on assumed flux and frequency distribution of the sources.

Depending on the shape of primordial bispectrum in consideration, some secondary bispectra are more dangerous than others. For example, ISW-lensing $B^{\mathrm{ISW}-\kappa}$ peaks at the "local" configurations hence, it is more dangerous for local primordial shape than the equilateral primordial shape. For example for the Planck satellite if the secondary bispectrum is not incorporated in the analysis, the ISW-lensing contribution will bias the estimate for the local $f_{\mathrm{NL}}$ by around $\Delta f_{\mathrm{NL}}^{\text {local }} \approx 10[19]$. The bispectrum contribution from primary-lensing-Rees-Sciama signal also peaks at squeezed limit and contribute to effective local $f_{\mathrm{NL}}^{\text {local }} \approx 10[132]$. 
For Planck sensitivity the point source will contamination the local non-Gaussianity by around $\Delta f_{\mathrm{NL}}^{\text {local }} \sim 1$ [133]. A recent analysis of the full second-order Boltzmann equation for photons [134] claims that second order effects add a contamination $\Delta f_{\mathrm{NL}} \sim 5$.

The generalization of the Fisher matrix given by (30) to include multiple bispectrum contribution is

$$
\begin{aligned}
\mathcal{F}_{a b}^{(X Y)}= & \sum_{\{i j k, \mathrm{pqr}\}} \sum_{\ell_{1} \leq \ell_{2} \leq \ell_{3}} \frac{1}{\Delta \ell_{1} \ell_{2} \ell_{3}} \frac{\partial B_{\ell_{1} \ell_{2} \ell_{3}}^{\mathrm{pqr},(X)}}{\partial p_{a}} \\
& \times\left(\mathrm{Cov}^{-1}\right)_{i j k, \mathrm{pqr}} \frac{\partial B_{\ell_{1} \ell_{2} \ell_{3}}^{i j k,(Y)}}{\partial p_{b}},
\end{aligned}
$$

where the additional $X$ and $Y$ indices denote a component such as primordial, point-sources, and ISW-lensing, and so forth. For fixed cosmological parameters, the signal to noise $(S / N)_{i}$ for the component $i$ is

$$
\left(\frac{S}{N}\right)_{i}=\frac{1}{\sqrt{\mathcal{F}^{(i i)}}} .
$$

\subsubsection{Non-Gaussianities from Recombination. Non-} Gaussianities can be generated during recombination. One requires to solve second-order Boltzmann and Einstein equations to evaluate the effect. The second-order effect on CMB is an active field of study [135-153] see [154] for a recent review. The non-Gaussianities produced during recombination comprise of various effects, for example, see [135].

The dominant bispectrum due to perturbative recombination comes from perturbations in the electron density. The amplitude of perturbations of the free electron density $\delta_{e}$ is around a factor of 5 larger than the baryon density perturbations [155]. The bispectrum generated due to $\delta_{e}$ peaks around the "local" configuration with corresponding effective non-linearity amplitude $f_{\mathrm{NL}} \sim$ few $[156,157]$.

The bispectrum contribution due to second-order terms which are the products of the first-order perturbations is calculated in [158]. The bispectrum contribution from these terms which also peak for the squeezed triangles is small and can be neglected in the analysis. For example, the signal to noise is about 0.4 at $l_{\max }=2000$ for a full-sky, cosmic variance limited experiment.

Another contribution to bispectrum which peaks for the equilateral configurations comes from the nonlinear evolution of the second order gravitational potential. Because of this effect, the minimum detectable non-Gaussianity parameter $f_{\mathrm{NL}}^{\text {equil. }}$ changes by $\Delta f_{\mathrm{NL}}^{\text {equil. }}=\mathcal{O}(10)$ for Planck-like experiment [126]. The bispectrum peaks for the equilateral shape because the growth of potential happens on scales smaller than the horizon size.

On large scales, in the absence of primordial nonGaussianities and assuming matter domination (so that the early and late ISW can be neglected), it has been shown in [151] that for the squeezed limit the effective $f_{\mathrm{NL}}$ generated by second-order gravitational effects on the $\mathrm{CMB} f_{\mathrm{NL}}=$ $-1 / 6-\cos (2 \theta)$ (also see $[141,152,153])$. Here $\theta$ is the angle between the short and the long modes. The angle dependent contribution comes from lensing.
5.3.3. Effect of Cosmological Parameter Uncertainties. Impact of uncertainties on the cosmological parameters effect, the error bar on $f_{\mathrm{NL}}$. The effect of cosmological parameters have been discussed in $[25,26,28,159]$. The cosmological parameters are determined using the 2-point statistics of the $\mathrm{CMB}$, and therefor we expect the largest effect of $f_{\mathrm{NL}}$ would come from those parameters which leave the $\mathrm{CMB}$ power spectrum unchanged while change the bispectrum. The expectation value of the estimator

$$
\left\langle\hat{f}_{\mathrm{NL}}\right\rangle=\frac{1}{N} \sum_{\ell_{1}, \ell_{2}, \ell_{3}} \frac{B_{\ell_{1} \ell_{2} \ell_{3}} \hat{B}_{\ell_{1} \ell_{2} \ell_{3}}}{C_{\ell_{1}} C_{\ell_{2}} C_{\ell_{3}}}
$$

changes with the change in cosmological parameters. Here $\widehat{B}_{\ell_{1} \ell_{2} \ell_{3}}$ is the true CMB bispectrum. When changing the parameters the normalization $N$ should be changed to make the estimator unbiased. In general for a set of cosmological paramerets $\left\{p_{i}\right\}$, the error in $f_{\mathrm{NL}}$ is given by [159]

$$
\delta \hat{f}_{\mathrm{NL}}=\sqrt{\left.\left.\sum_{i j} \frac{\partial f_{\mathrm{NL}}}{\partial p_{i}}\right|_{p_{i}=\bar{p}_{i}} \frac{\partial f_{\mathrm{NL}}}{\partial p_{j}}\right|_{p_{j}=\bar{p}_{j}} \operatorname{Cov}\left(p_{i}, p_{j}\right)} .
$$

Here the average parameter values $\bar{p}_{i}$ and their covariance matrix $\operatorname{Cov}\left(p_{i}, p_{j}\right)$ can be determined using CMB-likelihood analysis tools.

If the parameters are allowed to vary in the analysis then for WMAP this increases the $1 \sigma$ uncertainty in $f_{\mathrm{NL}}$ by $\delta f_{\mathrm{NL}}^{\text {local }} / f_{\mathrm{NL}} \approx 16 \%$ for the local shape and $\delta f_{\mathrm{NL}}^{\text {equil }} / f_{\mathrm{NL}} \approx$ $14 \%$ for the equilateral shape. For Planck experiment, the increases in $1 \sigma$ uncertainity are $\delta f_{\mathrm{NL}}^{\text {local }} / f_{\mathrm{NL}} \approx 5 \%$ for local shape and $\delta f_{\mathrm{NL}}^{\text {equil. }} / f_{\mathrm{NL}} \approx 4 \%$ for the equilateral shape. Most of the contribution to the error comes from three cosmological parameters, the amplitude of scalar perturbations $\Delta_{\Phi}$, the tilt of the power spectrum of the saclar perturbations $n_{S}$, and re-ionization optical depth $\tau$.

For modes inside the horizon during reionization, the reionization optical depth $\tau$ appears as a multiplicative factor $e^{-\tau}$ in front of transfer function $g_{\ell}^{i}$. For local model one of the mode is outside so the effect on bispectrum $\tilde{b}_{\ell_{1} \ell_{2} \ell_{3}}^{\text {local }}=\exp (-2 \tau) b_{\ell_{1} \ell_{2} \ell_{3}}^{\text {local }}$ and for equilateral model all the modes are inside the horizon so $\widetilde{b}_{\ell_{1} \ell_{2} \ell_{3}}^{\text {equil. }}=\exp (-3 \tau) b_{\ell_{1} \ell_{2} \ell_{3}}^{\text {equil. }}$. This reduces to $\delta f_{\mathrm{NL}}^{\text {local }} \simeq-2 f_{\mathrm{NL}} \tau$ for local model and $\delta f_{\mathrm{NL}}^{\text {equil. }} \simeq-3 f_{\mathrm{NL}} \tau$ for equilateral model.

The effect of amplitude of perturbations can be seen by noting that the level of non-Gaussianity is given by $f_{\mathrm{NL}} \cdot \Delta_{\Phi}^{1 / 2}$. Hence the decreases (increase) in the amplitude of perturbations relax (tighten) the constraints on $f_{\mathrm{NL}}$. The effect of red tilt $\left(n_{s}<1\right)$ can be thought of as a reduction in power on at scales shorter than first peak and enhancement of power on larger scales. The effect of blue tilt is just opposite of red tilt. For local shape, the limit on $f_{\mathrm{NL}}$ becomes tighter proportional to $\Delta_{\text {long }}^{1 / 2}[26]$. Note that [159] shows that the effect of cosmological parameters is negligible if the parameters are allowed to vary in the analysis and then marginalize over. 
5.3.4. Instrumental Effects and Distortions along the Line of Sight. Here we point out that any cosmological or instrumental effect that can be modelled as a line- of sight $\mathrm{CMB}$ distortions of the primary $\mathrm{CMB}$ doesnot generate new bispectrum contribution. Although they can modify the primordial bispectrum. A general model of line-of-sight distortions of the primary $\mathrm{CMB}$ are described in $[58,59$, 160], where the changes in the Stokes parameter of the CMB due to distortions along the line-of-sight can be written as

$$
\begin{aligned}
\delta[Q \pm i U](\hat{\mathbf{n}}) \\
=[\mathbf{a} \pm i 2 w](\widehat{\mathbf{n}})[\widetilde{Q} \pm i \widetilde{U}](\widehat{\mathbf{n}})+\left[f_{1} \pm i f_{2}\right](\widehat{\mathbf{n}})[\widetilde{Q} \mp i \tilde{U}](\widehat{\mathbf{n}}) \\
\quad+\left[\gamma_{1} \pm i \gamma_{2}\right](\widehat{\mathbf{n}}) \widetilde{T}(\widehat{\mathbf{n}})+\sigma p(\widehat{\mathbf{n}}) \cdot \nabla[\widetilde{Q} \pm i \widetilde{U}](\widehat{\mathbf{n}} ; \sigma) \\
\quad+\sigma\left[d_{1} \pm i d_{2}\right](\widehat{\mathbf{n}})\left[\partial_{1} \pm i \partial_{2}\right] \widetilde{T}(\widehat{\mathbf{n}} ; \sigma) \\
\quad+\sigma^{2} q(\widehat{\mathbf{n}})\left[\partial_{1} \pm i \partial_{2}\right]^{2} \widetilde{T}(\hat{\mathbf{n}} ; \sigma)+\cdots
\end{aligned}
$$

The first line captures the distortions in a single perfectly known direction $\hat{\mathbf{n}}$. The distortions in second line capture mixing of the polarization fields in a local region of length scale $\sigma$ around $\hat{\mathbf{n}}$. We Taylor expand the CMB fields $Q, U$, and $T$ around the point $\hat{\mathbf{n}}$ and consider the leading-order terms. Here $\widetilde{Q}, \tilde{U}$, and $\widetilde{T}$ stand for primordial (undistorted) CMB fields. Since $(Q \pm i U)(\hat{\mathbf{n}})$ is spin \pm 2 field, $a(\hat{\mathbf{n}})$ is a scalar field that describes modulation in the amplitude of the fields in a given direction $\widehat{\mathbf{n}} ; \omega(\widehat{\mathbf{n}})$ is also a scalar field that describes the rotation of the plane of polarization, $\left(f_{1} \pm i f_{2}\right)$ are spin \pm 4 fields that describe the coupling between two spin states (spin-flip), and $\left(\gamma_{1} \pm i \gamma_{2}\right)(\widehat{\mathbf{n}})$ are spin \pm 2 fields that describe leakage from the temperature to polarization (monopole leakage hereon). Distortions in the second line of (54), $\left(p_{1} \pm p_{2}\right),\left(d_{1} \pm d_{2}\right)$, and $q$ are measured in the units of the length scale $\sigma$. The field $\left(p_{1} \pm i p_{2}\right)(\widehat{\mathbf{n}})$ is a spin \pm 1 field and describes the change in the photon direction; we will refer to it as a deflection field. Finally $\left(d_{1} \pm d_{2}\right)(\widehat{\mathbf{n}})$ and $q(\widehat{\mathbf{n}})$ describe leakage from temperature to polarization, $\left(d_{1} \pm d_{2}\right)(\hat{\mathbf{n}})$ is spin \pm 1 field, and we will refer to it as dipole leakage; $q(\hat{\mathbf{n}})$ is a scalar field that we will call quadrupole leakage.

These distortions can be produced by various cosmological processes such as weak gravitational lensing of the $\mathrm{CMB}$, screening effects from patchy reionization, rotation of the plane of polarization due to magnetic fields or parity violating physics, and various instrumental systematics such as gain fluctuations, pixel rotation, differential gain, pointing, differential ellipticity are also captured via line-ofsight distortions. All these distortions modify the primordial bispectrum as

$$
\begin{aligned}
\widetilde{B}_{\left(\ell_{1}, \ell_{2}, \ell_{3}\right)} & \\
= & B_{\left(\ell_{1}, \ell_{2}, \ell_{3}\right)}+\int \frac{d^{2} \ell^{\prime}}{(2 \pi)^{2}} C_{l^{\prime}}^{\mathcal{D}} \\
& \times\left[B_{\left(\ell_{1}, \ell_{2}-\ell^{\prime}, \ell_{3}+\ell^{\prime}\right)} W^{\mathcal{D}}\left(\ell_{1}-\ell^{\prime}\right) W^{\mathcal{D}}\left(\ell_{2}-\ell^{\prime \prime}\right)+\text { perm. }\right]
\end{aligned}
$$

where $W$ is a window which depends on the type of distortion in consideration and tells how the primordial $\mathrm{CMB}$ bispectrum modes are coupled to the distortion field power spectrum $C_{\ell}^{\mathcal{D} D}$. The effect of the distortions on the bispectrum is to smooth out the acoustic features. These effects for the case of lensing have been shown to be small and can be neglected $[161,162]$.

In [163], the impact of the $1 / f$ noise and asymmetric beam on local $f_{\mathrm{NL}}^{\text {local }}$ has been found insignificant in the context of a Planck-like experiment.

\section{Other Probes of Non-Gaussianity in the CMB}

Although using the full bispectrum is the most sensitive cubic statistic other statistical methods may be sensitive to different aspects of non-Gaussianity and, more importantly, different methods have different systematic effects. Therefore it is important to study various probes. In this section we will discuss some of the methods which have been recently used or developed to test for primordial non-Gaussianities in the CMB.

Trispectrum. The four-point function in harmonic space is called trispectrum, which can be written as

$$
\begin{aligned}
& \left\langle a_{l_{1} m_{1}} a_{l_{2} m_{2}} a_{l_{3} m_{3}} a_{l_{4} m_{4}}\right\rangle \\
& \quad=\sum_{L M}(-1)^{M}\left(\begin{array}{ccc}
\ell_{1} & \ell_{2} & L \\
m_{1} & m_{2} & m_{3}
\end{array}\right)\left(\begin{array}{ccc}
e_{3} & \ell_{4} & L \\
m_{1} & m_{2} & m_{3}
\end{array}\right) T_{l_{3} l_{4}}^{l_{1} l_{2}}(L),
\end{aligned}
$$

where $T_{l_{3} l_{4}}^{l_{1} l_{2}}(L)$ is the angular averaged trispectrum, $L$ is the length of a diagonal that forms triangles with $l_{1}$ and $l_{2}$ and with $l_{3}$ and $l_{4}$, and the matrix is the Wigner 3-j symbol. The trispectrum contains unconnected part, $T_{G}$,

$$
\begin{aligned}
T_{G}^{l_{l_{3} l_{4}}} l_{2}(L)= & (-1)^{l_{1}+l_{3}} \sqrt{\left(2 l_{1}+1\right)\left(2 l_{3}+1\right)} C_{l_{1}} C_{l_{3}} \delta_{l_{1} l_{2}} \delta_{l_{3} l_{4}} \delta_{L 0} \\
& +(2 L+1) C_{l_{1}} C_{l_{2}}\left[(-1)^{l_{1}+l_{2}+L} \delta_{l_{1} l_{3}} \delta_{l_{2} l_{4}}+\delta_{l_{1} l_{4}} \delta_{l_{2} l_{3}}\right]
\end{aligned}
$$

which comes from the Gaussian part of the perturbations, and the connected part $T_{c}$ which contains non-Gaussian signatures. Using permutation symmetry, one may write the connected part of the trispectrum as

$$
\begin{aligned}
T_{c l_{3} l_{4}}^{l_{1} l_{2}}(L)= & P_{l_{3} l_{4}}^{l_{1} l_{2}}(L)+(2 L+1) \sum_{L^{\prime}} \\
& \times\left[(-1)^{l_{2}+l_{3}}\left\{\begin{array}{lll}
\ell_{1} & \ell_{2} & L \\
\ell_{4} & \ell_{3} & L^{\prime}
\end{array}\right\} P_{l_{2} l_{4}}^{l_{1} l_{3}}\left(L^{\prime}\right)\right. \\
& \left.+(-1)^{L+L^{\prime}}\left\{\begin{array}{lll}
\ell_{1} & \ell_{2} & L \\
\ell_{3} & \ell_{4} & L^{\prime}
\end{array}\right\} P_{l_{3} l_{2}}^{l_{1} l_{4}}\left(L^{\prime}\right)\right] .
\end{aligned}
$$


where

$$
\begin{aligned}
P_{l_{3} l_{4}}^{l_{1} l_{2}}(L)= & t_{l_{3} l_{4}}^{l_{1} l_{2}}(L)+(-1)^{2 L+l_{1}+l_{2}+l_{3}+l_{4}} t_{l_{4} l_{3}}^{l_{2} l_{1}}(L) \\
& +(-1)^{L+l_{3}+l_{4}} t_{l_{4} l_{3}}^{l_{1} l_{2}}(L)+(-1)^{L+l_{1}+l_{2}} t_{l_{3} l_{4}}^{l_{2} l_{1}}(L) .
\end{aligned}
$$

Here, the matrix is the Wigner $6-j$ symbol, and $t_{l_{3} l_{4}}^{l_{1} l_{2}}(L)$ is called the reduced trispectrum, which contains all the physical information about non-Gaussianities. For nonGaussianity of local-type for which

$$
\Phi(x)=\Phi_{L}(x)+f_{\mathrm{NL}}\left[\Phi_{L}^{2}-\left\langle\Phi_{L}^{2}\right\rangle\right]+g_{\mathrm{NL}} \Phi_{L}^{3},
$$

both $f_{\mathrm{NL}}$ and $g_{\mathrm{NL}}$ contribute to the trispectrum, but only $f_{\mathrm{NL}}$ contributes to the bispectrum. Tripectrum-based estimators for measuring $f_{\mathrm{NL}}$ and $g_{\mathrm{NL}}$ have been developed [164168]. For local template, the bispectrum nearly contains all the information on $f_{\mathrm{NL}}[166]$ however if the nonGaussianity is seen in bispectrum, trispectrum can serve as a important cross-check. Generically for single field slowroll models the trispectrum is small and unobservable [169] however for more general single field models whenever the equilateral bispectrum is large, the trispectrum is large as well [170-172]. For example for equilateral non-Gaussianity [173] study how to tune the model parameters to get large trispectrum and small bispectrum. For multifield inflation one can construct models that predicts small $f_{\mathrm{NL}}$ but large $g_{\mathrm{NL}}$, for example, [174] discussed the local form from a multi-field inflation and briefly mentioned the condition in their class of models to get the large trispectrum and small bispectrum. Joint constraints on both $f_{\mathrm{NL}}$ and $g_{\mathrm{NL}}$ have the potential to add to the specificity of the search for primordial non-Gaussianity. For a given model, these two numbers will often be predicted in terms of a single model parameter, such as a coupling constant see, for example, [175] for the case of ekyprotic models. Using WMAP 5-year data, the constraints on $g_{\mathrm{NL}}$ using the trispectrum are $-7.4<g_{\mathrm{NL}} / 10^{5}<8.2$ at $2 \sigma$ [176].

Minkowski Functionals. Minkowski Functionals (MFs) describe morphological properties (such as area, circumference, and Euler characteristic) of fluctuating fields [177-180]. For a $d$-dimensional fluctuating field, $f$, the $k$ th Minkowski Functionals of weakly non-Gaussian fields in, $V_{k}^{(d)}(\nu)$ for a given threshold $\nu=f / \sigma_{0}$ can be written as [181, 182]

$$
\begin{aligned}
& V_{k}^{(d)}(\nu) \\
& =\frac{1}{(2 \pi)^{(k+1) / 2}} \frac{\omega_{d}}{\omega_{d-k} \omega_{k}}\left(\frac{\sigma_{1}}{\sqrt{d} \sigma_{0}}\right)^{k} e^{-\nu^{2} / 2} \\
& \quad \times\left\{H_{k-1}(\nu)+\left[\frac{1}{6} S^{(0)} H_{k+2}(\nu)+\frac{k}{3} S^{(1)} H_{k}(\nu)\right.\right. \\
& \left.\left.\quad+\frac{k(k-1)}{6} S^{(2)} H_{k-2}(\nu)\right] \sigma_{0}+\mathcal{O}\left(\sigma_{0}^{2}\right)\right\},
\end{aligned}
$$

where $\sigma_{0} \equiv\left\langle f^{2}\right\rangle^{1 / 2}$ is the variance of the fluctuating field, $H_{n}(\nu)$ are the Hermite polynomials, $\omega_{k} \equiv \pi^{k / 2} / \Gamma(k / 2+1)$, and finally $S^{(i)}$ are the "skewness parameters" defined as

$$
\begin{gathered}
S^{(0)} \equiv \frac{\left\langle f^{3}\right\rangle}{\sigma_{0}^{4}}, \\
S^{(1)} \equiv-\frac{3}{4} \frac{\left\langle f^{2}\left(\nabla^{2} f\right)\right\rangle}{\sigma_{0}^{2} \sigma_{1}^{2}}, \\
S^{(2)} \equiv-\frac{3 d}{2(d-1)} \frac{\left\langle(\nabla f) \cdot(\nabla f)\left(\nabla^{2} f\right)\right\rangle}{\sigma_{1}^{4}},
\end{gathered}
$$

which characterize the skewness of fluctuating fields and their derivatives. Here $\sigma_{i}$ characterizes the variance of the fluctuating field and is given by

$$
\sigma_{i}^{2}=\frac{1}{4 \pi} \sum_{l}(2 \ell+1)[\ell(\ell+1)]^{i} C_{\ell}^{2} .
$$

For $\mathrm{CMB}$, for which $d=2$ and $f=\Delta T / T$, the skewness parameters are [183]

$$
\begin{aligned}
S^{(0)}= & \frac{1}{4 \pi \sigma_{0}^{4}} \sum_{l_{i} m_{i}} B_{l_{1} l_{2} l_{3}}^{m_{1} m_{2} m_{3}} g_{l_{1} l_{2} l_{3}}^{m_{1} m_{2} m_{3}} W_{l_{1}} W_{l_{2}} W_{l_{3}}, \\
S^{(1)}= & \frac{3}{16 \pi \sigma_{0}^{2} \sigma_{1}^{2}} \sum_{l_{i} m_{i}} \frac{l_{1}\left(l_{1}+1\right)+l_{2}\left(l_{2}+1\right)+l_{3}\left(l_{3}+1\right)}{3} \\
& \times B_{l_{1} l_{2} l_{3}}^{m_{1} m_{3} m_{3}} g_{l_{1} l_{2} l_{3} m_{3}}^{m_{1} m_{2} m_{l_{1}} W_{l_{2}} W_{l_{3}},} \\
S^{(2)}= & \frac{3}{8 \pi \sigma_{1}^{4}} \sum_{l_{i} m_{i}}\left\{\frac{\left[l_{1}\left(l_{1}+1\right)+l_{2}\left(l_{2}+1\right)-l_{3}\left(l_{3}+1\right)\right]}{3}\right. \\
& \times W_{l_{1}} W_{l_{2}} W_{l_{3}},
\end{aligned}
$$

where $B_{l_{1} l_{2} l_{3}}$ is the $\mathrm{CMB}$ bispectrum, $W_{l}$ represents a smoothing kernel which depends on the experiment beam and $g_{l_{1}, l_{2}, l_{3}}^{m_{1} m_{3}}$ is the usual Gaunt function.

Since MFs can be determined as weighted sum of the bispectrum, they contain less information than the bispectrum. MFs can still be useful because they perhaps suffer from different systematics, though they are less specific to primordial non-Gaussianity since they measure a smaller number of independent bispectrum modes. Also, the bispectrum is defined in Fourier (or harmonic) space while the MFs are defined in real space. Limits on non-Gaussianity of local-type from the MFs of the WMAP 5-year temperature data are $-70<f_{\mathrm{NL}}<91(2 \sigma)[188]$. The MFs from the Planck temperature data should be sensitive to $f_{\mathrm{NL}} \sim 20$ at $1 \sigma$ level [193] in contrast to bispectrum which is sensitive to $f_{\mathrm{NL}} \sim 5$ at $1 \sigma$ level. Note that polarization data further improves the sensitivity.

Wavelets. Several studies have used wavelet representations of the WMAP maps to search for a non-Gaussian signal 
TABLe 2: Summary of constraints on local non-Gaussianity.

\begin{tabular}{lcccc}
\hline Year & Data & Method & $f_{\mathrm{NL}}^{\text {local }} \pm 2 \sigma$ error & \\
\hline 2002 & COBE & Bispectrum sub-optimal & $\left|f_{\mathrm{NL}}\right|<1500$ & Komatsu et al. [184] \\
2003 & MAXIMA & Bispectrum sub-optimal & $\left|f_{\mathrm{NL}}\right|<1900$ & Santos et al. [185] \\
2003 & WMAP 1-year & Bispectrum sub-optimal & $39.5 \pm 97.5$ & Komatsu et al. [23] \\
2004 & VSA & Bispectrum sub-optimal & $f_{\mathrm{NL}}<5400$ & Smith et al. [186] \\
2005 & WMAP 1-year & Bispectrum sub-optimal-vl & $47 \pm 74$ & Creminelli et al. [25] \\
2006 & WMAP 3-year & Bispectrum sub-optimal & $30 \pm 84$ & Spergel et al. [24] \\
2006 & WMAP 3-year & Bispectrum sub-optimal-v1 & $32 \pm 68$ & Creminelli et al. [26] \\
2007 & WMAP 3-year & Bispectrum near-optimal & $87 \pm 62$ & Yadav and Wandelt [28] \\
2007 & Boomerang & Minkowski Functionals & $110 \pm 910$ & de Troia et al. [187] \\
2008 & WMAP 3-year & Minkowski Functionals & $51 \pm 60$ & Hikage et al. [188] \\
2008 & WMAP 5-year & Bispectrum near-optimal & $70_{-950}^{1075}$ & Komatsu et al. [48] \\
2008 & ARCHEOPS & Minkowski Functionals & $58 \pm 46$ & Curto et al. 2008 [189] \\
2009 & WMAP 3-year & Bispectrum optimal & $38 \pm 42$ & Smith et al. [122] \\
2009 & WMAP 5-year & Bispectrum optimal & $31 \pm 49$ & Smith et al. [122] \\
2009 & WMAP 5-year & Spherical Mexican hat wavelet & $-315 \pm 705$ & Curto, et al. [190] \\
2009 & BOOMERanG & Minkowski Functionals & $11 \pm 47.4$ & P. Natoli et al. [191] \\
2009 & WMAP 5-year & Skewness power spectrum & $32 \pm 42$ & Smidt, et al. [192] \\
2010 & WMAP 7-year & Bispectrum optimal & & Komatsu et al. [123] \\
\hline
\end{tabular}

[194-200]. In most of these studies, wavelets were used as a tool for blind searches of non-Gaussian anomalies in a basis with resolution in both scale and location. However, in some more recent studies, wavelets were tuned to look for non-Gaussianity of a particular type. In the context of searches for primordial non-Gaussianity of local type, wavelet-based estimators for $f_{\mathrm{NL}}$ have been built by extracting a signature of local non-Gaussianity that is cubic in the wavelet coefficients from simulations of non-Gaussian skies and searching for this signature in data. This ability to calibrate on a set of simulations makes the wavelet approach very flexible. While not optimal in a least-squared sense, using a wavelet representation can be thought of as a generalized cubic statistic with a different weighting scheme to the optimal bispectrum estimator. Using such estimators therefore provides a useful exploration of nearly optimal cubic estimators similar to the full bispectrum estimator. Any believable detection of non-Gaussianity should be robust to such changes in the analysis. Similarly, contaminating nonGaussianity from astrophysical and instrumental systematics will propagate through the analysis in a different way to the bispectrum-based analysis.

There are several constraints on local $f_{\mathrm{NL}}$ using waveletbased estimators. For example, using the COBE data the constraints are $\left|f_{\mathrm{NL}}\right|<2200(1 \sigma)[201]$. Using an estimator based on the skewness of the wavelet coefficients, Mukherjee and Wang constrain the $f_{\mathrm{NL}}$ value for WMAP 1-yr data obtaining $f_{\mathrm{NL}}=50 \pm 160(2 \sigma)$ [29]. Using an extension of the previous estimator by combining wavelet coefficients at different contiguous scales, Curto et al. obtain $-8<$ $f_{\mathrm{NL}}<111(2 \sigma)$ [202]. Recently, using a generalized thirdorder estimator based on the wavelet coefficients, Curto et al. obtain $-18<f_{\mathrm{NL}}<80(2 \sigma)$ [190].
Needlet Bispectrum. Needlets are a family of spherical wavelets which are localized and asymptotically uncorrelated $[203,204]$. The needlet-based statistics has been considered for testing Gaussianity and isotropy (see, e.g., [205-211]). Using the bispectrum of needlet coefficient, the constraints on non-Gaussianity of local-type using WMAP 5-year data yield $f_{\mathrm{NL}}=73 \pm 62(2 \sigma)[212,213]$. As is clear, the needletbased bispectrum is not as sensitive as the CMB bispectrum discussed in Section 4; however again in the event of detection the needlet based methods can be calibrated on simulations and represent a different weighting scheme for handling the sky mask and anisotropic noise. Finally, needlets and wavelets allow for the possibility to analyze spatially localized regions in the sky.

Probing Non-Gaussianity Using Bayesian Statistics. A somewhat different approach to searching for non-Gaussianity is provided by the Bayesian approach. Here, the starting point is an explicit physical or statistical model for the data and the goal is to evaluate the posterior density of the parameters of the model and/or the relative probability of the Gaussianity and non-Gaussianity.

On large scales, in the Sachs-Wolfe regime, one can simplify the Bayesian approach by modeling directly the temperature anisotropy. Rocha et al. (2001) [214] discuss a Bayesian exploration of a model, where each spherical harmonic coefficient is drawn from a non-Gaussian distribution. In this regime, the simple form of the non-Gaussian potential for the local model (17) also translates into a simple model for the temperature anisotropy. Reference [215] develop several results for it, including an analytical expression of the evidence ratio of the Gaussian and non-Gaussian models. At the level of current data, this approximation is too restrictive, 
since most of the information about $f_{\mathrm{NL}}$ is contained near the resolution limit of the experiment, where most of the measured perturbation modes are concentrated.

A full implementation of a physical non-Gaussian model must include the effect of Boltzmann transport. In the context of local non-Gaussianity, the model equation (17) suggests that a full Bayesian treatment may be feasible. At the time of writing, no fully Bayesian analysis for local $f_{\mathrm{NL}}$ has been published. The effort has focused on developing approximations to the full Bayesian problem.

Using a perturbative analysis, [216] relates the frequentist bispectrum estimator to moments of the Bayesian posterior distribution. Reference [217] described approximations to the full Bayesian treatment that simplify the analysis for high signal-to-noise maps and compared these to the full Bayesian treatment for a simple 1D toy model of non-Gaussian sky where this analysis is feasible.

\section{Summary}

The physics of the early universe responsible for generating the seed perturbations in the CMB is not understood. Inflation which is perhaps the the most promising paradigm for generating seed perturbations allows for vast number of inflationary models that are compatible with data based on 2-point statistics like CMB power spectrum. Moreover, the alternatives to inflation such as cyclic models are also compatible with the data. Characterizing the non-Gaussianity in the primordial perturbations has emerged as probe for discriminating between different models of the early universe. Models based on slowly rolling single field produce undetectable amount of non-Gaussianity. Single field models without the slow roll can generate large (detectable with future experiments) non-Gaussianities but (1) cannot produce large non-Gaussianity of local type unless inflation started with excited vacuum state, (2) if non-Gaussianity is produced it would naturally be as bispectrum while higher order as trispectrum can be generated, it requires fine tuning.

The bispectrum of the $\mathrm{CMB}$ is one of the most promising tool for connecting the non-Gaussianities in the cosmic microwave background and the models of inflation. Bispectrum-based estimator which saturates Cramer-Rao bound has been developed and well characterised using non-Gaussian MonteCarlos. Other statistics although not as sensitive to non-Gaussianity as an optimally weighted bispectrum estimator do provide independent checks and have different systematics. While Bayesian analysis has been applied in the context of nonGaussianity analysis, this still appears to be an open area for fruitful investigation.

Given the importance of detecting primordial nonGaussianity, it is crucial to characterise any nonprimordial sources of non-Gaussianities. We describe several sources of non-Gaussianities such as from second-order anisotropies after last scattering surface and during recombination.

With Planck launched and taking data, we look forward to the next few years as an exciting time in the exploration of primordial non-Gaussianity in the cosmic microwave background.

\section{Acknowledgment}

A. P.S. Yadav gratefully acknowledges support from the IBM Einstein Fellowship.

\section{Endnotes}

1. Although inflation is the most popular theory for the early universe, other mechanisms, for example, ekpyrotic models [218] and cyclic models [219, 220] have been proposed for generating nearly scale invariant Gaussian perturbations, while retaining homogeneity and flatness. In the cyclic universe, there is no beginning of time, and our expansion of the universe is one out of the infinite number of such cycles. Each cycle consists of the following phases. (1) A hot big bang phase, during which a structure formation takes place. (2) An accelerated expansion phase which dilutes the matter and radiation energy density. Since observations suggest that our universe is going through an accelerated expansion phase, in the cyclic model interpretation, we are presently going through this phase. (3) A decelerating phase, which makes the universe flat, and generates nearly Gaussian and scale invariant density perturbations. (4) A big crunch/bang transition phase during which matter and radiation is created. Although the mechanism is different, the outcome of phase (3) of the cyclic model is in some sense analogous to a slow-roll expansion phase of inflation; and phase (4) will correspond with the reheating phase in the inflationary scenario. As we will discuss in the next section these two scenarios can be distinguished by their different predictions about the gravitational waves, and non-Gaussianity. Cyclic models predict negligible contribution of gravitational waves while inflationary models can produce large gravitational wave contribution, which can be detected by next generation experiments. Second, cyclic models produce much larger non-Gaussianity (of local type) in comparison to the standard slow-roll inflationary scenario.

2. For the scale invariant $(n=1)$ case, $C_{l}^{S \mathrm{~W}}=$ $[l(l+1)]^{-1} 6 C_{2}^{\mathrm{SW}}$.

3. Example of some inflationary models are: eternal inflation, hybrid inflation, chaotic, Ghost inflation, Tilted Ghost inflation, DBI inflation, brane inflation, Nflation, bubble inflation, extended inflation, false vacuum inflation, power law inflation, k-inflation, hyperextended inflation, supersymmetric inflation, Quintessential inflation, Natural inflation, Super inflation, Supernatural inflation, D-term inflation, B -inflation, Thermal inflation, discrete inflation, Assisted inflation, Polar cap inflation, Open inflation, Topological inflation, Double inflation, Multiple inflation, Induced-gravity inflation, Warm inflation, stochastic inflation, Generalized assisted inflation, self-sustained inflation, Graduated inflation, Local inflation, Singular inflation, Slinky inflation, locked inflation, Elastic inflation, Mixed 
inflation, Phantom inflation, Boundary inflation, Noncommutative inflation, Tachyonic inflation, Tsunami inflation, Lambda inflation, Steep inflation, Oscillating inflation, Mutated Hybrid inflation, intermediate inflation, and Inhomogeneous inflation.

4. To first order in perturbations, primordial scalar perturbations do not generate B-modes of CMB. However at second (and higher) order in perturbations, scalar perturbations do produce B-modes $[135,221]$. The B-modes generated from higher order perturbations are expected to be smaller than the tensor B-mode levels that the upcoming and future experiments (like $\mathrm{CMBPol}$ ) are sensitive too.

5. Or equivalently $\Phi(\mathbf{r})=\Phi_{L}(\mathbf{r})+f_{\mathrm{NL}}\left(\Phi_{L}^{2}(\mathbf{r})-\left\langle\Phi_{L}^{2}(\mathbf{r})\right\rangle\right)$.

6. We will refer to the estimator in [25] as a near-optimalv1, while the corrected estimator of [28] as nearoptimal.

\section{References}

[1] A. H. Guth, "Inflationary universe: a possible solution to the horizon and flatness problems," Physical Review D, vol. 23, no. 2, pp. 347-356, 1981.

[2] K. Sato, "Cosmological baryon-number domain structure and the first order phase transition of a vacuum," Physics Letters B, vol. 99, no. 1, pp. 66-70, 1981.

[3] A. D. Linde, "A new inflationary universe scenario: a possible solution of the horizon, flatness, homogeneity, isotropy and primordial monopole problems," Physics Letters B, vol. 108, no. 6, pp. 389-393, 1982.

[4] A. Albrecht and P. J. Steinhardt, "Cosmology for grand unified theories with radiatively induced symmetry breaking," Physical Review Letters, vol. 48, no. 17, pp. 1220-1223, 1982.

[5] A. H. Guth and S. -Y. Pi, "Fluctuations in the new inflationary universe," Physical Review Letters, vol. 49, no. 15, pp. 1110 $1113,1982$.

[6] A. A. Starobinsky, "Dynamics of phase transition in the new inflationary universe scenario and generation of perturbations," Physics Letters B, vol. 117, no. 3-4, pp. 175-178, 1982.

[7] S. W. Hawking, "The development of irregularities in a single bubble inflationary universe," Physics Letters B, vol. 115, no. 4, pp. 295-297, 1982.

[8] J. M. Bardeen, P. J. Steinhardt, and M. S. Turner, "Spontaneous creation of almost scale-free density perturbations in an inflationary universe," Physical Review D, vol. 28, no. 4, pp. 679-693, 1983.

[9] V. F. Mukhanov, H. A. Feldman, and R. H. Brandenberger, "Theory of cosmological perturbations," Physics Report, vol. 215, no. 5-6, pp. 203-333, 1992.

[10] D. S. Salopek and J. R. Bond, "Nonlinear evolution of long-wavelength metric fluctuations in inflationary models," Physical Review D, vol. 42, no. 12, pp. 3936-3962, 1990.

[11] D. S. Salopek and J. R. Bond, "Stochastic inflation and nonlinear gravity," Physical Review D, vol. 43, no. 4, pp. 10051031, 1991.

[12] T. Falk, R. Rangarajan, and M. Srednicki, "The angular dependence of the three-point correlation function of the cosmic microwave background radiation as predicted by inflationary cosmologies," Astrophysical Journal, vol. 403, no. 1, pp. L1-L3, 1993.
[13] A. Gangui, F. Lucchin, S. Matarrese, and S. Mollerach, "The three-point correlation function of the cosmic microwave background in inflationary models," Astrophysical Journal, vol. 430, no. 2, pp. 447-457, 1994.

[14] V. Acquaviva, N. Bartolo, S. Matarrese, and A. Riotto, "Gauge-invariant second-order perturbations and nonGaussianity from inflation,” Nuclear Physics B, vol. 667, no. 1-2, pp. 119-148, 2003.

[15] J. Maldacena, "Non-gaussian features of primordial fluctuations in single field inflationary models," Journal of High Energy Physics, vol. 7, no. 5, pp. 233-264, 2003.

[16] N. Bartolo, E. Komatsu, S. Matarrese, and A. Riotto, "NonGaussianity from inflation: theory and observations," Physics Reports, vol. 402, no. 3-4, pp. 103-266, 2004.

[17] E. Komatsu, D. N. Spergel, and B. D. Wandelt, "Measuring primordial non-Gaussianity in the cosmic microwave background," Astrophysical Journal, vol. 634, no. 1, pp. 14-19, 2005.

[18] P. Cabella, F. K. Hansen, M. Liguori et al., "The integrated bispectrum as a test of cosmic microwave background nonGaussianity: detection power and limits on fNL with WMAP data," Monthly Notices of the Royal Astronomical Society, vol. 369, no. 2, pp. 819-824, 2006.

[19] K. M. Smith and M. Zaldarriaga, " Algorithms for bispectra: forecasting, optimal analysis, and simulation," http://arxiv.org/abs/astro-ph/0612571.

[20] A. P. S. Yadav, E. Komatsu, B. D. Wandelt, M. Liguori, F. K. Hansen, and S. Matarrese, "Fast estimator of primordial nonGaussianity from temperature and polarization anisotropies in the cosmic microwave background. II. Partial sky coverage and inhomogeneous noise," Astrophysical Journal, vol. 678, no. 2, pp. 578-582, 2008.

[21] A. P. S. Yadav, E. Komatsu, and B. D. Wandelt, "Fast estimator of primordial non-Gaussianity from temperature and polarization anisotropies in the cosmic microwave background," Astrophysical Journal, vol. 664, no. 2, pp. 680686, 2007.

[22] E. Komatsu, B. D. Wandelt, D. N. Spergel, A. J. Banday, and K. M. Górski, "Measurement of the cosmic microwave background bispectrum on the COBE DMR sky maps," Astrophysical Journal, vol. 566, no. 1, pp. 19-29, 2002.

[23] E. Komatsu, A. Kogut, M. R. Nolta et al., "First-year Wilkinson Microwave Anisotropy Probe (WMAP) observations: tests of Gaussianity," Astrophysical Journal, Supplement Series, vol. 148, no. 1, pp. 119-134, 2003.

[24] D. N. Spergel, R. Bean, O. Dore' et al., “Three-year Wilkinson Microwave Anisotropy Probe (WMAP) observations: implications for cosmology," Astrophysical Journal, Supplement Series, vol. 170, no. 2, pp. 377-408, 2007.

[25] P. Creminelli, A. Nicolis, L. Senatore, M. Tegmark, and M. Zaldarriaga, "Limits on non-Gaussianities from WMAP data," Journal of Cosmology and Astroparticle Physics, no. 5, article 4, 2006.

[26] P. Creminelli, L. Senatore, M. Zaldarriaga, and M. Tegmark, "Limits on f_NL parameters from WMAP 3yr data," Journal of Cosmology and Astro-Particle Physics, vol. 3, article 5, 2007.

[27] G. Chen and I. Szapudi, "Constraining primordial nongaussianities from the WMAP2 2-1 cumulant correlator power spectrum," Astrophysical Journal, vol. 647, no. 2, pp. L87-L90, 2006.

[28] A. P. S. Yadav and B. D. Wandelt, "Evidence of primordial non-gaussianity ( $\mathrm{fNL}$ ) in the wilkinson microwave

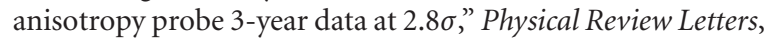
vol. 100, no. 18, Article ID 181301, 2008. 
[29] P. Mukherjee and Y. Wang, "Wavelets and Wilkinson microwave anisotropy probe non-gaussianity," Astrophysical Journal, vol. 613, no. 1, pp. 51-60, 2004.

[30] D. L. Larson and B. D. Wandelt, "The hot and cold spots in the Wilkinson Microwave Anisotropy Probe data are not hot and cold enough," Astrophysical Journal, vol. 613, no. 2, pp. L85-L88, 2004.

[31] P. Vielva, E. Gonzalez, R. B. Barreiro, J. L. Sanz, and L. Cayón, "Detection of non-gaussianity in the Wilkinson microwave anisotropy probe first-year data using spherical wavelets," Astrophysical Journal, vol. 609, no. 1, pp. 22-34, 2004.

[32] L.-Y. Chiang, P. D. Naselsky, O. V. Verkhodanov, and M. J. Way, "Non-Gaussianity of the derived maps from the first-year Wilkinson Microwave Anisotropy Probe data," Astrophysical Journal, vol. 590, no. 2, pp. L65-L68, 2003.

[33] L.-Y. Chiang, P. D. Naselsky, and P. Coles, "The robustness of phase mapping as a non-Gaussianity test," Astrophysical Journal, vol. 602, no. 1, pp. L1-L4, 2004.

[34] D. Babich and M. Zaldarriaga, "Primordial bispectrum information from CMB polarization," Physical Review D, vol. 70, no. 8, Article ID 083005, 2004.

[35] A. P. S. Yadav and B. D. Wandelt, "CMB tomography: reconstruction of adiabatic primordial scalar potential using temperature and polarization maps," Physical Review D, vol. 71, no. 12, Article ID 123004, 2005.

[36] J. M. Kovac, E. M. Leitch, C. Pryke, J. E. Carlstrom, N. W. Halverson, and W. L. Holzapfel, "Detection of polarization in the cosmic microwave background using DASI," Nature, vol. 420, no. 6917, pp. 772-787, 2002.

[37] A. Kogut, D. N. Spergel, C. Barnes et al., "First-year Wilkinson Microwave Anisotropy Probe (WMAP) observations: temperature-polarization correlation," Astrophysical Journal, Supplement Series, vol. 148, no. 1, pp. 161-173, 2003.

[38] L. Page, G. Hinshaw, E. Komatsu et al., "Three-year Wilkinsonmicrowave Anisotropy Probe (WMAP) observations: polarization analysis," Astrophysical Journal, Supplement Series, vol. 170, no. 2, pp. 335-376, 2007.

[39] T. E. Montroy, P. A. R. Ade, J. J. Bock et al., "A Measurement of the CMB (EE) Spectrum from the 2003 flight of BOOMERANG," Astrophysical Journal, vol. 647, no. 2, pp. 813-822, 2006.

[40] D. Baumann, “TASI lectures on inflation," http://arxiv.org/ abs/0907.5424.

[41] V. F. Mukhanov, H. A. Feldman, and R. H. Brandenberger, "Theory of cosmological perturbations," Physics Report, vol. 215, no. 5-6, pp. 203-333, 1992.

[42] W. A. Bardeen, R. B. Pearson, and E. Rabinovici, "Hadron masses in quantum chromodynamics on the transverse lattice," Physical Review D, vol. 21, no. 4, pp. 1037-1054, 1980.

[43] H. Kodama and M. Sasaki, "Cosmological perturbation theory," Progress of Theoretical Physics Supplement, no. 78, 1984.

[44] R. K. Sachs and A. M. Wolfe, "Perturbations of a cosmological model and angular variations of the microwave background," The Astrophysical Journal, vol. 147, p. 73, 1967.

[45] J. R. Bond and G. Efstathiou, "The statistics of cosmic background radiation fluctuations," Monthly Notices of the Royal Astronomical Society, vol. 226, pp. 655-687, 1987.

[46] P. J. E. Peebles and J. T. Yu, "Primeval adiabatic perturbation in an expanding universe," The Astrophysical Journal, vol. 162, pp. 815-836, 1970.
[47] U. Seljak and M. Zaldarriaga, "A line-of-sight integration approach to cosmic microwave background anisotropies," Astrophysical Journal, vol. 469, no. 2, pp. 437-444, 1996.

[48] E. Komatsu, J. Dunkley, M. R. Nolta et al., "Five-year Wilkinson Microwave Anisotropy Probe (WMAP) observations: cosmological interpretation," The Astrophysical Journal Supplement Series, vol. 180, no. 2, pp. 330-376, 2009.

[49] D. Baumann, M. G. Jackson, P. Adshead et al., " Probing inflation with CMB polarization," in Proceedings of the CMB Polarization Workshop. Theory and Foregrounds: CMBPol Mission Concept, vol. 1141 of AIP Conference Proceedings, pp. 10-120, 2009.

[50] B. P. Abbott, R. Abbott, F. Acernese et al., "An upper limit on the stochastic gravitational-wave background of cosmological origin," Nature, vol. 460, no. 7258, pp. 990-994, 2009.

[51] U. Seijak and M. Zaldarriaga, "Signature of gravity waves in the polarization of the microwave background," Physical Review Letters, vol. 78, no. 11, pp. 2054-2057, 1997.

[52] M. Kamionkowski, A. Kosowsky, and A. Stebbins, "Statistics of cosmic microwave background polarization," Physical Review D, vol. 55, no. 12, pp. 7368-7388, 1997.

[53] M. Kamionkowski, A. Kosowsky, and A. Stebbins, "A probe of primordial gravity waves and vorticity," Physical Review Letters, vol. 78, no. 11, pp. 2058-2061, 1997.

[54] ACTPol, BICEP, CAPMAP, CBI, Clover, CMBPol, EBEX, POLARBEAR, PIPER, QUaD et al., current and upcomming experimets.

[55] U. Seljak, "Gravitational lensing effect on cosmic microwave background anisotropies: a power spectrum approach," Astrophysical Journal, vol. 463, no. 1, pp. 1-7, 1996.

[56] M. Zaldarriaga and U. Seljak, "Gravitational lensing effect on cosmic microwave background polarization," Physical Review D, vol. 58, no. 2, Article ID 023003, 1998.

[57] A. P. S. Yadav, R. Biswas, M. Su, and M. Zaldarriaga, "Constraining a spatially dependent rotation of the cosmic microwave background polarization," Physical Review D, vol. 79, no. 12, Article ID 123009, 2009.

[58] W. Hu, M. M. Hedman, and M. Zaldarriaga, "Benchmark parameters for CMB polarization experiments," Physical Review D, vol. 67, no. 4, Article ID 043004, 2003.

[59] A. P. S. Yadav, M. Su, and M. Zaldarriaga, "Primordial Bmode diagnostics and self-calibrating the CMB polarization," Physical Review D, vol. 81, no. 6, Article ID 063512, 2010.

[60] A. D. Linde, "Generation of isothermal density perturbations in an inflationary universe," JETP Letters, vol. 40, pp. 13331336, 1984.

[61] L. A. Kofman and A. D. Linde, "Generation of density perturbations in inflationary cosmology," Nuclear Physics B, vol. 282, pp. 555-588, 1987.

[62] D. Polarski and A. A. Starobinsky, "Isocurvature perturbations in multiple inflationary models," Physical Review D, vol. 50, no. 10, pp. 6123-6129, 1994.

[63] C. Gordon, D. Wands, B. A. Bassett, and R. Maartens, "Adiabatic and entropy perturbations from inflation," Physical Review D, vol. 63, no. 2, Article ID 023506, 2001.

[64] A. D. Linde, "Generation of isothermal density perturbations in the inflationary universe," Physics Letters B, vol. 158, no. 5, pp. 375-380, 1985.

[65] G. Efstathiou and J. R. Bond, "Isocurvature cold dark matter fluctuations," Monthly Notices of the Royal Astronomical Society, vol. 218, pp. 103-121, 1986. 
[66] P. J. E. Peebles, "Origin of the large-scale galaxy peculiar velocity field: a minimal isocurvature model," Nature, vol. 327, no. 6119, pp. 210-211, 1987.

[67] H. Kodama and M. Sasaki, "Evolution of isocurvature perturbations I: Photon-baryon universe," International Journal of Modern Physics A, vol. 1, no. 1, pp. 265-301, 1986.

[68] S. Weinberg, "Can nonadiabatic perturbations arise after single-field inflation?" Physical Review D, vol. 70, no. 4, Article ID 043541, 2004.

[69] J. Garcia-Bellido and D. Wands, "Metric perturbations in two-field inflation," Physical Review D, vol. 53, no. 10, pp. 5437-5445, 1996.

[70] M. Sasaki and E. D. Stewart, "A general analytic formula for the spectral index of the density perturbations produced during inflation," Progress of Theoretical Physics, vol. 95, no. 1, pp. 71-78, 1996.

[71] M. Sasaki and T. Tanaka, "Super-horizon scale dynamics of multi-scalar inflation," Progress of Theoretical Physics, vol. 99, no. 5, pp. 763-781, 1998.

[72] N. Bartolo, S. Matarrese, and A. Riotto, "Adiabatic and isocurvature perturbations from inflation: power spectra and consistency relations," Physical Review D, vol. 64, no. 12, Article ID 123504, 2001.

[73] M. Bucher, K. Moodley, and N. Turok, "Characterizing the primordial cosmic perturbations using MAP and Planck," Physical Review D, vol. 66, no. 2, Article ID 023528, 2002.

[74] M. Bucher, K. Moodley, and N. Turok, "Constraining isocurvature perturbations with cosmic microwave background polarization," Physical Review Letters, vol. 87, no. 19, Article ID 191301, 4 pages, 2001.

[75] R. Bean, J. Dunkley, and E. Pierpaoli, "Constraining isocurvature initial conditions with WMAP 3-year data," Physical Review D, vol. 74, no. 6, Article ID 063503, 2006.

[76] S. Gupta, A. Berera, A. F. Heavens, and S. Matarrese, "NonGaussian signatures in the cosmic background radiation from warm inflation," Physical Review D, vol. 66, no. 4, Article ID 043510, 2002.

[77] T. J. Allen, B. Grinstein, and M. B. Wise, "Non-gaussian density perturbations in inflationary cosmologies," Physics Letters B, vol. 197, no. 1-2, pp. 66-70, 1987.

[78] J. Lesgourgues, D. Polarski, and A. A. Starobinsky, "Quantum-to-classical transition of cosmological perturbations for non-vacuum initial states," Nuclear Physics B, vol. 497, no. 1-2, pp. 479-508, 1997.

[79] J. Martin, A. Riazuelo, and M. Sakellariadou, "Nonvacuum initial states for cosmological perturbations of quantummechanical origin," Physical Review D, vol. 61, no. 8, Article ID 083518, pp. 1-15, 2000.

[80] P. Creminelli, "On non-Gaussianities in single-field inflation," Journal of Cosmology and Astroparticle Physics, no. 10, pp. 53-62, 2003.

[81] N. Arkani-Hamed, P. Creminelli, S. Mukohyama, and M. Zaldarriaga, "Ghost inflation," Journal of Cosmology and Astroparticle Physics, no. 4, pp. 1-18, 2004.

[82] G. Dvali, A. Gruzinov, and M. Zaldarriaga, "New mechanism for generating density perturbations from inflation," Physical Review D, vol. 69, no. 2, Article ID 023505, 2003.

[83] M. Zaldarriaga, "Non-Gaussianities in models with a varying inflaton decay rate," Physical Review D, vol. 69, no. 4, Article ID 043508, 2004.

[84] D. H. Lyth, C. Ungarelli, and D. Wands, "Primordial density perturbation in the curvaton scenario," Physical Review D, vol. 67, no. 2, Article ID 023503, 2003.
[85] E. Komatsu, N. Afshordi, N. Bartolo et al., "Non-Gaussianity as a probe of the physics of the primordial universe and the astrophysics of the low redshift universe," http://arxiv.org/ abs/0902.4759.

[86] D. Babich, P. Creminelli, and M. Zaldarriaga, "The shape of non-Gaussianities," Journal of Cosmology and Astroparticle Physics, no. 8, pp. 199-217, 2004.

[87] N. Bartolo, S. Matarrese, and A. Riotto, "Non-Gaussianity from inflation," Physical Review D, vol. 65, no. 10, Article ID 103505, 2002.

[88] F. Bernardeau and J.-P. Uzan, "Non-Gaussianity in multifield inflation," Physical Review D, vol. 66, Article ID 103506, 2002.

[89] F. Bernardeau and J.-P. Uzan, "Inflationary models inducing non-Gaussian metric fluctuations," Physical Review D, vol. 67, no. 12, Article ID 121301, 2003.

[90] M. Sasaki, "Multi-brid inflation and non-gaussianity," Progress of Theoretical Physics, vol. 120, no. 1, pp. 159-174, 2008.

[91] A. Naruko and M. Sasaki, "Large non-Gaussianity from multi-brid inflation," Progress of Theoretical Physics, vol. 121, no. 1, pp. 193-210, 2009.

[92] C. T. Byrnes, K.-Y. Choi, and L. M. H. Hall, "Conditions for large non-Gaussianity in two-field slow-roll inflation," Journal of Cosmology and Astroparticle Physics, vol. 2008, no. 10, article 8, 2008.

[93] C. T. Byrnes and D. Wands, "Curvature and isocurvature perturbations from two-field inflation in a slow-roll expansion," Physical Review D, vol. 74, no. 4, Article ID 043529, 2006.

[94] D. Langlois, F. Vernizzi, and D. Wands, "Non-linear isocurvature perturbations and non-Gaussianities," Journal of Cosmology and Astroparticle Physics, vol. 2008, no. 12, article 4, 2008.

[95] J. Valiviita, H. Assadullahi, and D. Wands, "Primordial nongaussianity from multiple curvaton decay," http://arxiv.org/ abs/0806.0623.

[96] H. Assadullahi, J. Väliviita, and D. Wands, "Primordial nonGaussianity from two curvaton decays," Physical Review D, vol. 76, no. 10, Article ID 103003, 2007.

[97] J. Valiviita, M. Sasaki, and D. Wands, "Non-Gaussianity and constraints for the variance of perturbations in the curvaton model," http://arxiv4.library.cornell.edu/abs/astro$\mathrm{ph} / 0610001$.

[98] F. Vernizzi and D. Wands, "Non-Gaussianities in two-field inflation," Journal of Cosmology and Astroparticle Physics, vol. 2006, no. 5, article 19, 2006.

[99] L. E. Allen, S. Gupta, and D. Wands, "Non-Gaussian perturbations from multi-field inflation," Journal of Cosmology and Astroparticle Physics, vol. 2006, no. 1, pp. 93-107, 2006.

[100] A. Linde and V. Mukhanov, "Non-Gaussian isocurvature perturbations from inflation," Physical Review D, vol. 56, no. 2, pp. R535-R539, 1997.

[101] L. Kofman, "Probing string theory with modulated cosmological fluctuations," http://arxiv.org/abs/astro-ph/0303614.

[102] J.-L. Lehners and P. J. Steinhardt, "Non-Gaussian density fluctuations from entropically generated curvature perturbations in ekpyrotic models," Physical Review D, vol. 77, no. 6, Article ID 063533, 2008.

[103] J.-L. Lehners and P. J. Steinhardt, "Intuitive understanding of non-Gaussianity in ekpyrotic and cyclic models," Physical Review D, vol. 78, no. 2, Article ID 023506, 2008.

[104] K. Koyama, S. Mizuno, and D. Wands, "Curvature perturbations from ekpyrotic collapse with multiple fields," Classical and Quantum Gravity, vol. 24, no. 15, pp. 3919-3931, 2007. 
[105] R. Holman and A. J. Tolley, "Enhanced non-Gaussianity from excited initial states," Journal of Cosmology and Astroparticle Physics, vol. 2008, no. 5, article 1, 2008.

[106] X. Chen, M.-X. Huang, S. Kachru, and G. Shiu, "Observational signatures and non-Gaussianities of general singlefield inflation," Journal of Cosmology and Astroparticle Physics, no. 1, article 2, 2007.

[107] L. Verde, L. Wang, A. F. Heavens, and M. Kamionkowski, "Large-scale structure, the cosmic microwave background and primordial non-Gaussianity," Monthly Notices of the Royal Astronomical Society, vol. 313, no. 1, pp. 141-147, 2000.

[108] E. Komatsu and D. N. Spergel, "Acoustic signatures in the primary microwave background bispectrum," Physical Review D, vol. 63, no. 6, Article ID 063002, 2001.

[109] K. Koyama, S. Mizuno, F. Vernizzi, and D. Wands, "NonGaussianities from ekpyrotic collapse with multiple fields," Journal of Cosmology and Astroparticle Physics, no. 11, pp. 121, 2007.

[110] E. I. Buchbinder, J. Khoury, and B. A. Ovrut, "NonGaussianities in new ekpyrotic cosmology," Physical Review Letters, vol. 100, no. 17, Article ID 171302, 2008.

[111] J. Maldacena, "Non-Gaussian features of primordial fluctuations in single field. Inflationary models," Journal of High Energy Physics, vol. 2003, no. 5, article 13, 2003.

[112] M. Alishahiha, E. Silverstein, and D. Tong, "DBI in the sky: non-Gaussianity from inflation with a speed limit," Physical Review D, vol. 70, no. 12, Article ID 123505, 2004.

[113] P. Creminelli, "On non-Gaussianities in single-field inflation," Journal of Cosmology and Astroparticle Physics, no. 10, pp. 53-62, 2003.

[114] D. Seery and J. E. Lidsey, "Primordial non-Gaussianities in single-field inflation," Journal of Cosmology and Astroparticle Physics, no. 6, pp. 45-70, 2005.

[115] C. Cheung, P. Creminelli, A. L. Fitzpatrick, J. Kaplan, and L. Senatore, "The effective field theory of inflation," Journal of High Energy Physics, vol. 2008, no. 3, article 14, 2008.

[116] P. D. Meerburg, J. P. van der Schaar, and P. S. Corasaniti, "Signatures of initial state modifications on bispectrum statistics," Journal of Cosmology and Astroparticle Physics, vol. 2009, no. 5, article 18, 2009.

[117] A. Penzias and R. Wilson, "A measurement of excess antenna temperature at $4080 \mathrm{Mc} / \mathrm{S}$," The Astrophysical Journal, vol. 142, pp. 419-421, 1965.

[118] G. F. Smoot, C. L. Bennett, A. Kogut et al., "Structure in the COBE differential microwave radiometer first-year maps," Astrophysical Journal, vol. 396, no. 1, pp. L1-L5, 1992.

[119] A. Lewis, A. Challinor, and A. Lasenby, "Efficient computation of cosmic microwave background anisotropies in closed Friedmann-Robertson-Walker models," Astrophysical Journal, vol. 538, no. 2, pp. 473-476, 2000.

[120] L. Senatore, K. M. Smith, and M. Zaldarriaga, "NonGaussianities in single field inflation and their optimal limits from the WMAP 5-year data," Journal of Cosmology and Astroparticle Physics, vol. 2010, no. 1, article 28, 2010.

[121] J. R. Fergusson and E. P.S. Shellard, "Shape of primordial non-Gaussianity and the CMB bispectrum," Physical Review D, vol. 80, no. 4, Article ID 043510, 2009.

[122] K. M. Smith, L. Senatore, and M. Zaldarriaga, "Optimal limits on fNLlocal from WMAP 5-year data," Journal of Cosmology and Astroparticle Physics, vol. 2009, no. 9, article 6, 2009.

[123] E. Komatsu, K. M. Smith, J. Dunkley et al., "Seven-year Wilkinson Microwave Anisotropy Probe (WMAP) observations: cosmological interpretation," http://arxiv.org/ abs/1001.4538.
[124] P. Creminelli, L. Senatore, and M. Zaldarriaga, "Estimators for local non-Gaussianities," Journal of Cosmology and Astroparticle Physics, no. 3, article 19, 2007.

[125] M. Liguori, A. Yadav, F. K. Hansen, E. Komatsu, S. Matarrese, and B. Wandelt, "Temperature and polarization CMB maps from primordial non-Gaussianities of the local type," Physical Review D, vol. 76, no. 10, Article ID 105016, 2007.

[126] N. Bartolo and A. Riotto, "On the non-Gaussianity from recombination," Journal of Cosmology and Astroparticle Physics, vol. 2009, no. 3, article 17, 2009.

[127] E. Sefusatti, M. Liguori, A. P. S. Yadav, M. G. Jackson, and E. Pajer, "Constraining running non-gaussianity," Journal of Cosmology and Astroparticle Physics, vol. 2009, no. 12, article 22, 2009.

[128] P. Serra and A. Cooray, "Impact of secondary nonGaussianities on the search for primordial non-Gaussianity with CMB maps," Physical Review D, vol. 77, no. 10, Article ID 107305, 2008.

[129] D. M. Goldberg and D. N. Spergel, "Microwave background bispectrum. II. A probe of the low redshift universe," Physical Review D, vol. 59, no. 10, Article ID 103002, 6 pages, 1999.

[130] D. N. Spergel and D. M. Goldberg, "Microwave background bispectrum. I. Basic formalism,” Physical Review D, vol. 59, no. 10, Article ID 103001, 8 pages, 1999.

[131] A. Cooray and W. Hu, "Imprint of reionization on the cosmic microwave background bispectrum," Astrophysical Journal, vol. 534, no. 2, pp. 533-550, 2000.

[132] A. Mangilli and L. Verde, "Non-Gaussianity and the CMB bispectrum: confusion between primordial and lensingRees-Sciama contribution?" Physical Review D, vol. 80, no. 12, Article ID 123007, 2009.

[133] D. Babich and E. Pierpaoli, "Point source contamination in CMB non-Gaussianity analyses," Physical Review D, vol. 77, no. 12, Article ID 123011, 2008.

[134] C. Pitrou, J. Uzan, and F. Bernardeau, "The cosmic microwave background bispectrum from the non-linear evolution of the cosmological perturbations," http://arxiv .org/abs/1003.0481.

[135] N. Bartolo, S. Matarrese, and A. Riotto, "CMB anisotropies at second-order II: analytical approach," Journal of Cosmology and Astroparticle Physics, no. 1, article 19, 2007.

[136] W. Hu, D. Scott, and J. Silk, "Reionization and cosmic microwave background distortions: a complete treatment of second-order Compton scattering," Physical Review D, vol. 49, no. 2, pp. 648-670, 1994.

[137] S. Dodelson and J. M. Jubas, "Reionization and its imprint on the cosmic microwave background," Astrophysical Journal, vol. 439, no. 2, pp. 503-516, 1995.

[138] T. Pyne and S. M. Carroll, "Higher-order gravitational perturbations of the cosmic microwave background," Physical Review D, vol. 53, no. 6, pp. 2920-2929, 1996.

[139] S. Mollerach and S. Matarrese, "Cosmic microwave background anisotropies from second order gravitational perturbations," Physical Review D, vol. 56, no. 8, pp. 4494-4502, 1997.

[140] S. Matarrese, S. Mollerach, and M. Bruni, "Relativistic second-order perturbations of the Einstein-de Sitter universe," Physical Review D, vol. 58, no. 4, Article ID 043504, 1998.

[141] P. Creminelli and M. Zaldarriaga, "CMB 3-point functions generated by nonlinearities at recombination," Physical Review D, vol. 70, no. 8, Article ID 083532, 2004. 
[142] N. Bartolo, S. Matarrese, and A. Riotto, "Gauge-invariant temperature anisotropies and primordial non-gaussianity," Physical Review Letters, vol. 93, no. 23, Article ID 231301, 2004.

[143] K. Tomita, "Relativistic second-order perturbations of nonzero- $\Lambda$ flat cosmological models and CMB anisotropies," Physical Review D, vol. 71, no. 8, Article ID 083504, 11 pages, 2005.

[144] N. Bartolo, S. Matarrese, and A. Riotto, "Non-Gaussianity of large-scale cosmic microwave background anisotropies beyond perturbation theory," Journal of Cosmology and Astroparticle Physics, no. 8, pp. 177-196, 2005.

[145] N. Bartolo, S. Matarrese, and A. Riotto, "The full secondorder radiation transfer function for large-scale $\mathrm{CMB}$ anisotropies," Journal of Cosmology and Astroparticle Physics, vol. 2006, no. 5, article 10, 2006.

[146] K. Tomita, "Second-order power spectra of CMB anisotropies due to primordial random perturbations in flat cosmological models," Physical Review D, vol. 77, no. 10, Article ID 103521, 2008.

[147] C. Pitrou, J.-P. Uzan, and F. Bernardeau, "Cosmic microwave background bispectrum on small angular scales," Physical Review D, vol. 78, no. 6, Article ID 063526, 2008.

[148] N. Bartolo, S. Matarrese, and A. Riotto, "Cosmic microwave background anisotropies at second order: I," Journal of Cosmology and Astroparticle Physics, vol. 2006, no. 6, article 24, 2006

[149] C. Pitrou, "The radiative transfer at second order: a full treatment of the Boltzmann equation with polarization," Classical and Quantum Gravity, vol. 26, no. 6, Article ID 065006, 2009.

[150] L. Senatore, S. Tassev, and M. Zaldarriaga, "Cosmological perturbations at second order and recombination perturbed," Journal of Cosmology and Astroparticle Physics, vol. 2009, no. 8, article 31, 2009.

[151] L. Boubekeur, P. Creminelli, G. D’Amico, J. Noreña, and F. Vernizzi, "Sachs-Wolfe at second order: the CMB bispectrum on large angular scales," Journal of Cosmology and Astroparticle Physics, vol. 2009, no. 8, article 29, 2009.

[152] N. Bartolo, S. Matarrese, and A. Riotto, "Enhancement of non-gaussianity after inflation," Journal of High Energy Physics, vol. 8, no. 4, pp. 147-159, 2004.

[153] N. Bartolo, S. Matarrese, and A. Riotto, "Evolution of secondorder cosmological perturbations and non-Gaussianity," Journal of Cosmology and Astroparticle Physics, vol. 2004, no. 1, pp. 47-70, 2004.

[154] N. Bartolo, S. Matarrese, and A. Riotto, "Non-Gaussianity and the cosmic microwave background anisotropies," http://arxiv.org/abs/1001.3957.

[155] B. Novosyadlyj, "Perturbations of ionization fractions at the cosmological recombination epoch," Monthly Notices of the Royal Astronomical Society, vol. 370, no. 4, pp. 1771-1782, 2006.

[156] R. Khatri and B. D. Wandelt, "Crinkles in the last scattering surface: non-Gaussianity from inhomogeneous recombination," Physical Review D, vol. 79, no. 2, Article ID 023501, 2009.

[157] L. Senatore, S. Tassev, and M. Zaldarriaga, "Nongaussianities from perturbing recombination," Journal of Cosmology and Astroparticle Physics, vol. 2009, no. 9, article 38, 2009.

[158] D. Nitta, E. Komatsu, N. Bartolo, S. Matarrese, and A. Riotto, "CMB anisotropies at second order III: bispectrum from products of the first-order perturbations," Journal of Cosmology and Astroparticle Physics, vol. 2009, no. 5, article 14, 2009.
[159] M. Liguori and A. Riotto, "Impact of uncertainties in the cosmological parameters on the measurement of primordial non-Gaussianity," Physical Review D, vol. 78, no. 12, Article ID 123004, 2008.

[160] M. Su, A. P. S. Yadav, and M. Zaldarriaga, "Impact of instrumental systematic contamination on the lensing mass reconstruction using the CMB polarization," Physical Review D, vol. 79, no. 12, Article ID 123002, 2009.

[161] D. Hanson, K. M. Smith, A. Challinor, and M. Liguori, "CMB lensing and primordial non-Gaussianity," Physical Review D, vol. 80, no. 8, Article ID 083004, 2009.

[162] A. Cooray, D. Sarkar, and P. Serra, "Weak lensing of the primary CMB bispectrum," Physical Review D, vol. 77, no. 12, Article ID 123006, 2008.

[163] S. Donzelli, F. K. Hansen, M. Liguori, and D. Maino, "Impact of the $1 / \mathrm{f}$ noise and the asymmetric beam on non-gaussianity searches with planck," Astrophysical Journal, vol. 706, no. 2, pp. 1226-1240, 2009.

[164] T. Okamoto and W. Hu, "Angular trispectra of CMB temperature and polarization," Physical Review D, vol. 66, no. 6, Article ID 063008, 2002.

[165] N. Kogo and E. Komatsu, "Angular trispectrum of CMB temperature anisotropy from primordial non-Gaussianity with the full radiation transfer function," Physical Review D, vol. 73, no. 8, Article ID 083007, 2006.

[166] P. Creminelli, L. Senatore, and M. Zaldarriaga, "Estimators for local non-Gaussianities," Journal of Cosmology and Astroparticle Physics, vol. 2007, no. 3, article 19, 2007.

[167] D. Munshi, A. Heavens, A. Cooray et al., "New optimised estimators for the primordial trispectrum," http://arxiv.org/ abs/0910.3693.

[168] D. M. Regan, E. P. S. Shellard, and J. R. Fergusson, "General CMB and primordial trispectrum estimation," http://arxiv.org/ abs/1004.2915.

[169] D. Seery, J. E. Lidsey, and M. S. Sloth, "The inflationary trispectrum," Journal of Cosmology and Astroparticle Physics, no. 1, article 27, 2007.

[170] M.-X. Huang and G. Shiu, "Inflationary trispectrum for models with large non-Gaussianities," Physical Review D, vol. 74, no. 12, article 121301, 2006.

[171] X. Chen, B. Hu, M.-X. Huang, G. Shiu, and Y. Wang, "Large primordial trispectra in general single field inflation," Journal of Cosmology and Astroparticle Physics, vol. 2009, no. 8, article 8, 2009.

[172] F. Arroja, S. Mizuno, K. Koyama, and T. Tanaka, "Full trispectrum in single field DBI inflation," Physical Review D, vol. 80, no. 4, Article ID 043527, 2009.

[173] K. T. Engel, K. S. M. Lee, and M. B. Wise, "Trispectrum versus bispectrum in single-field inflation," Physical Review D, vol. 79, no. 10, Article ID 103530, 2009.

[174] C. T. Byrnes, M. Sasaki, and D. Wands, "Primordial trispectrum from inflation," Physical Review D, vol. 74, no. 12, Article ID 123519, 2006.

[175] J.-L. Lehners and P. J. Steinhardt, "Non-Gaussianity generated by the entropic mechanism in bouncing cosmologies made simple," Physical Review D, vol. 80, no. 10, Article ID 103520, 2009.

[176] J. Smidt, A. Amblard, A. Cooray et al., "A measurement of cubic-order primordial non-Gaussianity (gnl and taunl) with WMAP 5-year data," http://arxiv.org/abs/1001.5026.

[177] K. R. Mecke, T. Buchert, and H. Wagner, "Robust morphological measures for large-scale structure in the universe," Astronomy and Astrophysics, vol. 288, pp. 697-704, 1994. 
[178] J. Schmalzing and T. Buchert, "Beyond genus statistics: a unifying approach to the morphology of cosmic structure," Astrophysical Journal, vol. 482, no. 1, pp. L1-L4, 1997.

[179] J. Schmalzing and K. M. Górski, "Minkowski functionals used in the morphological analysis of cosmic microwave background anisotropy maps," Monthly Notices of the Royal Astronomical Society, vol. 297, no. 2, pp. 355-365, 1998.

[180] S. Winitzki and A. Kosowsky, "Minkowski functional description of microwave background Gaussianity," New Astronomy, vol. 3, no. 2, pp. 75-99, 1998.

[181] T. Matsubara, "Analytic expression of the genus in a weakly non-Gaussian field induced by gravity," Astrophysical Journal, vol. 434, no. 2, pp. L43-L46, 1994.

[182] T. Matsubara, "Statistics of smoothed cosmic fields in perturbation theory. I. Formulation and useful formulae in second-order perturbation theory," Astrophysical Journal, vol. 584, no. 1, pp. 1-33, 2003.

[183] C. Hikage, E. Komatsu, and T. Matsubara, "Primordial nongaussianity and analytical formula for minkowski functionals of the cosmic microwave background and large-scale structure," Astrophysical Journal, vol. 653, no. 1, pp. 11-26, 2006.

[184] E. Komatsu, B. D. Wandelt, D. N. Spergel, A. J. Banday, and K. M. Górski, "Measurement of the cosmic microwave background bispectrum on the COBE DMR sky maps," Astrophysical Journal, vol. 566, no. 1, pp. 19-29, 2002.

[185] M. G. Santos, A. Heavens, A. Balbi et al., "Multiple methods for estimating the bispectrum of the cosmic microwave background with application to the MAXIMA data," Monthly Notices of the Royal Astronomical Society, vol. 341, no. 2, pp. 623-643, 2003.

[186] S. Smith, G. Rocha, A. Challinor et al., "Estimating the bispectrum of the very small array data," Monthly Notices of the Royal Astronomical Society, vol. 352, no. 3, pp. 887-902, 2004.

[187] G. de Troia, P. A. R. Ade, J. J. Bock et al., "Searching for non-Gaussian signals in the BOOMERanG 2003 CMB map: preliminary results," New Astronomy Reviews, vol. 51, no. 3-4, pp. 250-255, 2007.

[188] C. Hikage, T. Matsubara, P. Coles, M. Liguori, F. K. Hansen, and S. Matarrese, "Limits on primordial non-Gaussianity from Minkowski Functionals of the WMAP temperature anisotropies," Monthly Notices of the Royal Astronomical Society, vol. 389, no. 3, pp. 1439-1446, 2008.

[189] A. Curto, J.F. Macías-Pérez, E. Martínez-González et al., "Constraints on the non-linear coupling parameter fnl with Archeops data," Astronomy and Astrophysics, vol. 486, no. 2, pp. 383-391, 2008.

[190] A. Curto, E. Martínez-González, and R. B. Barreiro, "Improved constraints on primordial non-gaussianity for the wilkinson microwave anisotropy probe 5-year data," Astrophysical Journal, vol. 706, no. 1, pp. 399-403, 2009.

[191] P. Natoli, G. De Troia, C. Hikage et al., "BOOMERanG constraints on primordial non-Gaussianity from analytical minkowski functionals," http://arxiv.org/abs/0905.4301.

[192] J. Smidt, A. Amblard, P. Serra, and A. Cooray, "Measurement of primordial non-Gaussianity using the WMAP 5-year temperature skewness power spectrum," Physical Review D, vol. 80, no. 12, Article ID 123005, 2009.

[193] C. Hikage, E. Komatsu, and T. Matsubara, "Primordial nongaussianity and analytical formula for minkowski functionals of the cosmic microwave background and large-scale structure," Astrophysical Journal, vol. 653, no. 1, pp. 11-26, 2006.

[194] N. Aghanim and O. Forni, "Searching for the non-Gaussian signature of the CMB secondary anisotropies," Astronomy and Astrophysics, vol. 347, no. 2, pp. 409-418, 1999.
[195] M. P. Hobson, A. W. Jones, and A. N. Lasenby, "Wavelet analysis and the detection of non-Gaussianity in the cosmic microwave background," Monthly Notices of the Royal Astronomical Society, vol. 309, no. 1, pp. 125-140, 1999.

[196] P. Vielva, E. Martínez-González, R. B. Barreiro, J. L. Sanz, and L. Cayón, "Detection of non-gaussianity in the Wilkinson microwave anisotropy probe first-year data using spherical wavelets," Astrophysical Journal, vol. 609, no. 1, pp. 22-34, 2004.

[197] J. -L. Starck, N. Aghanim, and O. Forni, "Detection and discrimination of cosmological non-gaussian signatures by multi-scale methods," Astronomy and Astrophysics, vol. 416, no. 1, pp. 9-17, 2004.

[198] J. Jin, J.-L. Starck, D. L. Donoho, N. Aghanim, and O. Forni, "Cosmological non-Gaussian signature detection: comparing performance of different statistical tests," EURASIP Journal on Applied Signal Processing, vol. 2005, no. 15, pp. 2470-2485, 2005.

[199] P. Vielva, Y. Wiaux, E. Martínez-González, and P. Vandergheynst, "Steerable wavelet analysis of $\mathrm{CMB}$ structures alignment," New Astronomy Reviews, vol. 50, no. 11-12, pp. 880-888, 2006.

[200] J. D. McEwen, P. Vielva, Y. Wiaux et al., "Cosmological applications of a wavelet analysis on the sphere," Journal of Fourier Analysis and Applications, vol. 13, no. 4, pp. 495-510, 2007.

[201] L. Cayón, E. Martínez-González, F. Argüeso, A. J. Banday, and K. M. Górski, "COBE-DMR constraints on the nonlinear coupling parameter: a wavelet based method," Monthly Notices of the Royal Astronomical Society, vol. 339, no. 4, pp. 1189-1194, 2003.

[202] A. Curto, E. Martínez-González, P. Mukherjee et al., "Wilkinson microwave anisotropy probe 5-yr constraints on fnl with wavelets," Monthly Notices of the Royal Astronomical Society, vol. 393, no. 2, pp. 615-622, 2009.

[203] F. J. Narcowich, P. Petrushev, and J. D. Ward, "Localized tight frames on spheres," SIAM Journal on Mathematical Analysis, vol. 38, no. 2, pp. 574-594, 2006.

[204] F. Narcowich, P. Petrushev, and J. Ward, "Decomposition of Besov and Triebel-Lizorkin spaces on the sphere," Journal of Functional Analysis, vol. 238, no. 2, pp. 530-564, 2006.

[205] P. Baldi, G. Kerkyacharian, D. Marinucci, and D. Picard, "High frequency asymptotics for wavelet-based tests for Gaussianity and isotropy on the torus," Journal of Multivariate Analysis, vol. 99, no. 4, pp. 606-636, 2008.

[206] P. Baldi, G. Kerkyacharian, D. Marinucci, and D. Picard, "Asymptotics for spherical needlets," Annals of Statistics, vol. 37, no. 3, pp. 1150-1171, 2009.

[207] P. Baldi, G. Kerkyacharian, D. Marinucci, and D. Picard, "Subsampling needlet coefficients on the sphere," Bernoulli, vol. 15, pp. 438-463, 2009.

[208] X. Lan and D. Marinucci, "The needlets bispectrum," Electronic Journal of Statistics, vol. 2, pp. 332-367, 2008.

[209] D. Pietrobon, P. Cabella, A. Balbi, G. De Gasperis, and N. Vittorio, "Constraints on primordial non-Gaussianity from a needlet analysis of the WMAP-5 data," Monthly Notices of the Royal Astronomical Society, vol. 396, no. 3, pp. 1682-1688, 2009.

[210] D. Pietrobon, P. Cabella, A. Balbi, R. Crittenden, G. de Gasperis, and N. Vittorio, "Needlet bispectrum asymmetries in the WMAP 5-year data," Monthly Notices of the Royal Astronomical Society, vol. 402, p. L34, 2010. 
[211] P. Cabella, D. Pietrobon, M. Veneziani et al., "Foreground influence on primordial non-Gaussianity estimates: needlet analysis of WMAP 5-year data," Monthly Notices of the Royal Astronomical Society, vol. 504, pp. 961-968, 2010.

[212] $\varnothing$. Rudjord, F. K. Hansen, X. Lan, M. Liguori, D. Marinucci, and S. Matarrese, "An estimate of the primordial nongaussianity parameter $\mathrm{f}$ NL using the needlet bispectrum from wmap," Astrophysical Journal, vol. 701, no. 1, pp. 369-376, 2009.

[213] $\varnothing$. Rudjord, F. K. Hansen, X. Lan, M. Liguori, D. Marinucci, and S. Matarrese, "Directional variations of the nongaussianity parameter f NL," Astrophysical Journal, vol. 708, no. 2, pp. 1321-1325, 2010.

[214] G. Rocha, J. Magueijo, M. Hobson, and A. Lasenby, "Bayesian joint estimation of non-Gaussianity and the power spectrum," Physical Review D, vol. 64, no. 6, Article ID 063512, 2001.

[215] P. Vielva and J. L. Sanz, "Analysis of non-Gaussian CMB maps based on the N-pdf. Application to WMAP data," http://arxiv.org/abs/0812.1756.

[216] T. A. Enßlin, M. Frommert, and F. S. Kitaura, "Information field theory for cosmological perturbation reconstruction and non-linear signal analysis," Physical Review D, vol. 80, Article ID 105005, 2009.

[217] F. Elsner, B. D. Wandelt, and M. D. Schneider, "Probing local non-Gaussianities within a Bayesian framework," Astronomy and Astrophysics, vol. 513, no. 11, 2010.

[218] J. Khoury, B. A. Ovrut, P. J. Steinhardt, and N. Turok, "Ekpyrotic universe: colliding branes and the origin of the hot big bang," Physical Review D, vol. 64, no. 12, Article ID 123522, 2001.

[219] P. J. Steinhardt and N. Turok, "A cyclic model of the universe," Science, vol. 296, no. 5572, pp. 1436-1439, 2002.

[220] P. J. Steinhardt and N. Turok, "Cosmic evolution in a cyclic universe," Physical Review D, vol. 65, no. 12, Article ID 126003, 2002.

[221] D. Baumann, P. Steinhardt, K. Takahashi, and K. Ichiki, "Gravitational wave spectrum induced by primordial scalar perturbations," Physical Review D, vol. 76, no. 8, Article ID 084019, 2007. 

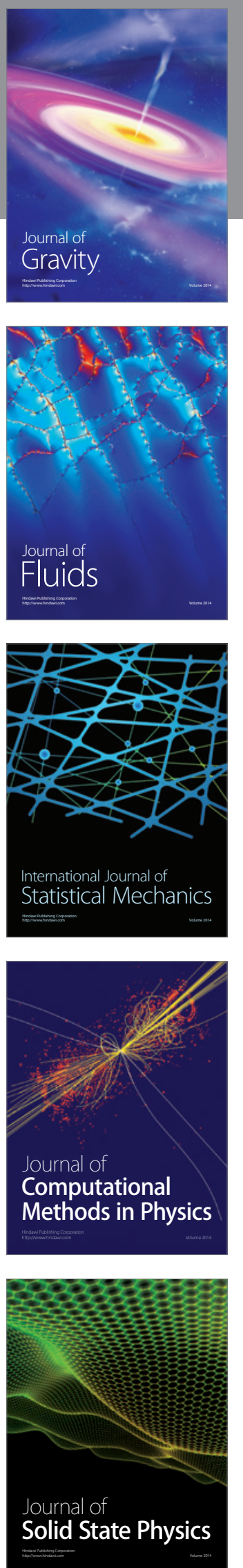

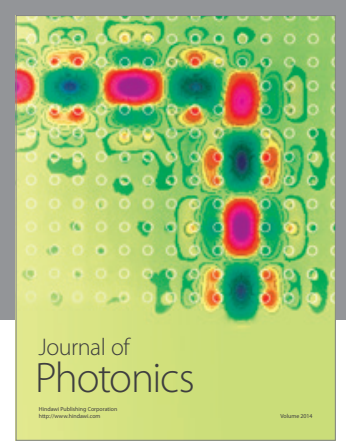

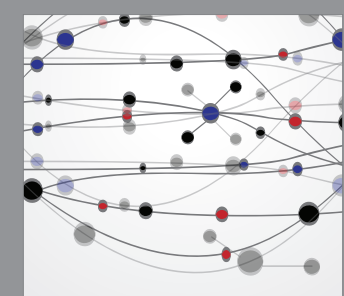

The Scientific World Journal
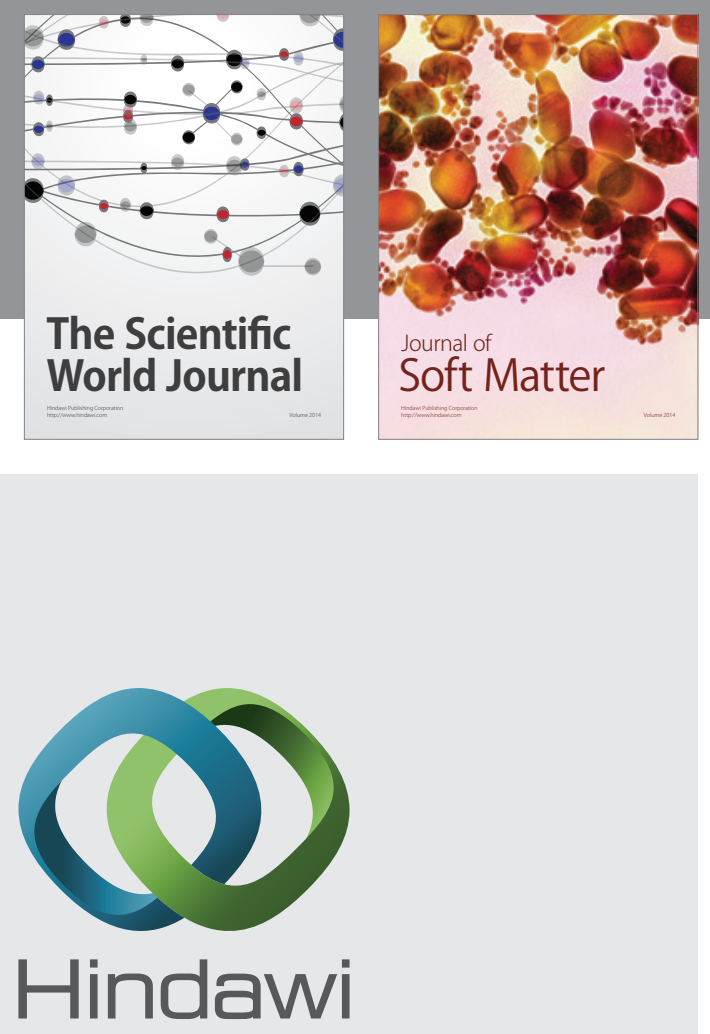

Submit your manuscripts at

http://www.hindawi.com
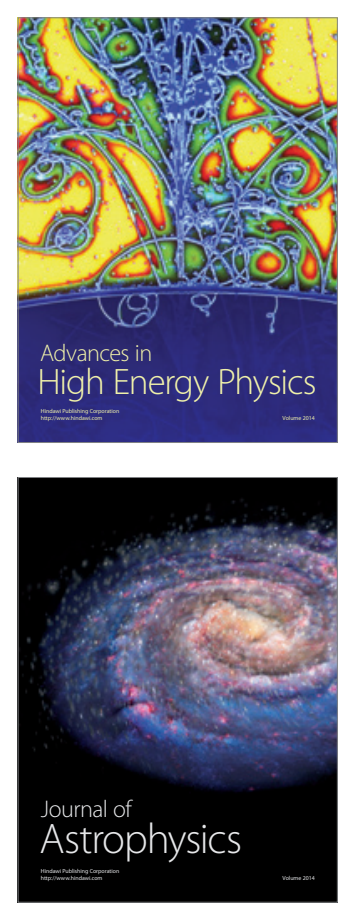
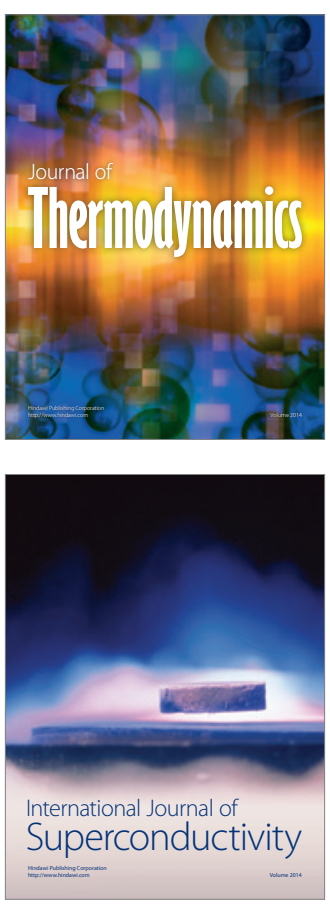
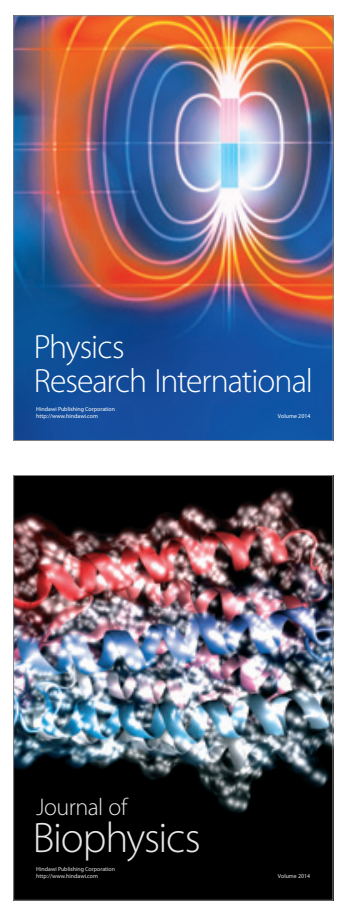
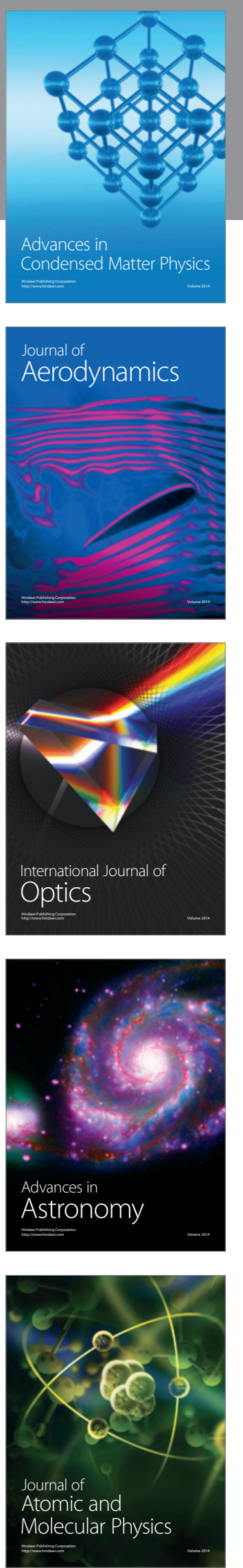